\title{
Conditional probabilistic estimates of 21st century greenhouse gas emissions based on the storylines of the IPCC-SRES scenarios
}

\author{
Detlef P. van Vuuren $^{\mathrm{a}, *}$, Bert de Vries ${ }^{\mathrm{a}}$, Arthur Beusen ${ }^{\mathrm{a}}$, Peter S.C. Heuberger ${ }^{\mathrm{b}}$ \\ a Netherlands Environmental Assessment Agency, PO Box 303, 3720 AH Bilthoven, The Netherlands \\ ${ }^{\mathrm{b}}$ Delft Center for Systems and Control, Delft University of Technology, The Netherlands
}

\section{A R T I C L E I N F O}

\section{Article history:}

Received 24 August 2007

Received in revised form

26 May 2008

Accepted 3 June 2008

\section{Keywords:}

Scenarios

Uncertainty

Greenhouse gases

Climate change

Probability

\begin{abstract}
A B S T R A C T
The conditional probabilistic scenario analysis combines statistical methods of uncertainty analysis at parameter level with storylines which recognize the deep uncertainty that exists for several underlying trends. The model calculations indicate that cumulative 21st century emissions could range from 800 to $2500 \mathrm{GtC}$ in the absence of climate policy. This range originates partly from the underlying storylines, and partly from the probabilistic analysis. Among the most important parameters contributing to the uncertainty range are uncertainty in income growth, population growth, parameters determining energy demand, oil resources and fuel preferences. The contribution of these factors is also scenario-dependent.
\end{abstract} (c) 2008 Elsevier Ltd. All rights reserved.

\section{Introduction}

Indications of possible long-term trends in the global energy system provide very essential information for policy makers. The energy system is by far the single most important driver of anthropogenic climate change, and also plays an important role in connection with several other sustainability problems such as regional air pollution and resource depletion. The future of the energy system is, however, beset with uncertainty, as it is the product of complex dynamic processes and factors, including demographic and economic development, technological change, energy policies and resource availability. Various development patterns for each of them could introduce very different futures for the energy system as a whole. Scenarios are tools used in the assessment of future developments of these complex systems that are either inherently unpredictable or characterized by large scientific uncertainties. In exploring future development of energy systems and climate change, uncertainty management therefore needs to be a constant companion of scientists and decisionmakers (Hulme and Carter, 1999). Uncertainty has various causes, varying from stochastic randomness to limitations in knowledge, and ignorance and human anticipation. Uncertainty can occur on different scales: model parameters, model structures and/or complete disagreement in conceptualization among experts (see next Section 2). The question how to deal with uncertainty in model projections has, in recent years, been given considerable attention (Grübler and Nakicenovic, 2001; Schneider, 2001, 2002; Webster et al., 2002; Patt and Dessai, 2005; Dessai et al., 2007). Two approaches are most prominent in the debate on handling uncertainty in the context of climate and (energy) emissions scenarios: (storyline-based) alternative scenarios and fully probabilistic scenarios.

The alternative scenarios approach is founded on the premise that possible future developments can vary over a large and partly unknown range, among other based on fundamentally different views of the current and desired situation (De Vries, 2006). These ranges are only partly bound by relationships among variables (so-called stylized facts ${ }^{1}$ ). Usually (energy) models endogenize only a limited number of these relationships as they may be too complex to incorporate and/or lack quantitative evidence. In the scenario approach, such relationships are expressed in a "storyline"; this storyline represents a kind of underlying logic of the scenario and its main assumptions. This way of providing consistency to the complex parts of the real-world developments forces modelers (and users) to think in a more creative way about possible future developments.

The fully probabilistic approach to uncertainties expresses the most important model inputs in terms of probability estimates

\footnotetext{
* Corresponding author. Tel.: +31302742046; fax: +31302744464.

E-mail address: detlef.van.vuuren@mnp.nl (D.P. van Vuuren).
}

\footnotetext{
${ }^{1}$ The term "stylized facts" refers to stable patterns that emerge from many different sources of empirical data.
} 
and uses statistical sampling techniques to create a range of emission pathways defined by a median value and various probability intervals. This approach is easily applicable to systems that are clearly defined and for which input parameters can be meaningfully expressed in terms of likelihood. The approach has also been applied to more complex systems, as in the modeling of future greenhouse gas trajectories (Webster et al., 2002, 2003). One might say that the fully probabilistic approach operates from the positivist engineering/control paradigm (the system is known well enough to make meaningful probabilistic estimates), whereas the alternative scenario approach positions itself more in a constructivist social science tradition (one can create alternative visions of the future, but without assigning likelihood) (De Vries, 2006).

The ongoing discussion between proponents of the individual approaches has revealed strengths and weaknesses of both approaches (see Section 2.1). The methods can, in our view, best be seen as complementary, not exclusive. In fact, one could also combine the two methods by simultaneously accepting ignorance for some aspects of future development, while at the same time bringing in elements of formal uncertainty analysis. O'Neill (2004, 2005) introduced such a "conditional probability approach" for population scenarios, with as rationale that is more meaningful to make judgments about the likelihood of future trends in the context of a particular development path, than about the likelihood of this path itself. While O'Neill applied this approach successfully in population scenarios, hardly any attempt has, so far, been made to use a similar approach for the total energy system.

The main focus of this paper is to explore what kind of information can be provided by a conditional probabilistic approach to uncertainty. For this purpose we have applied such an analysis using statistically sampled simulations of the TIMER energy model (van Vuuren et al., 2006a) conditional to the storylines of the IPCCSRES scenarios. We focus here, in particular, on one crucial output variable of this model, i.e. global $\mathrm{CO}_{2}$ emissions.

The aim was to provide insight into the following questions:

1. What range of emissions would result from a probabilistic approach to uncertainty?

2. What elements of uncertainty contribute most to these emission ranges?

3. How do results of a conditional probabilistic approach compare to other approaches of uncertainty?

Obviously, the answers to these questions depend on the modeling tool applied. A more complete account of uncertainties would be achieved by including more than one model (Nakicenovic and Swart, 2000; van Vuuren et al., 2006b). However, even then, some of the uncertainties will not be captured by any of the models. In the next section, we will discuss in more detail the value added and limitations of the conditional scenario. In brief, we feel that this article adds to existing literature by: (1) contributing to a discussion of methods for characterizing uncertainty in future emissions, (2) providing estimates of the influence of uncertainty in parameters, and (3) discussing the relationship of parameter uncertainty and storylines. The paper will contribute to but clearly not be the last word in the debate how different methods for uncertainty analysis can be used.

\section{Methods}

\subsection{Sources of uncertainty and earlier applications of uncertainty methods in scenario approaches}

Uncertainty originates from various causes and can be classified in different ways (NRC, 1996; Rotmans and de Vries,
1997; Moss and Schneider, 2000; Dessai and Hulme, 2001, 2004; Van der Sluijs et al., 2003; Patt and Dessai, 2005). One classification is based on the nature of the uncertainty (NRC, 1996; Petersen, 2006). Ontic uncertainty (a) refers to natural randomness, which can generally be expressed in mean estimates and their ranges of likelihood (for instance, uncertainty originating from chaotic behavior in complex systems). This type of uncertainty cannot be easily reduced. Its influence can sometimes be empirically determined (e.g. distribution of extreme weather events), although there is no guarantee that the same distribution will hold in the future. Epistemic uncertainty (b), in contrast, comes from incomplete knowledge (for instance, ultimately available oil resources). In the case of energy scenarios, an important part of the uncertainty originates from not knowing how the technoeconomic and socio-cultural context of the energy systems evolves. There are various subcategories of epistemic uncertainty based on the way it is handled (mostly subjective) statistical expressions (b1); conditional statements (b2) or recognized ignorance (b3). A special form of epistemic uncertainty comes from (c) disagreements among experts (Patt, 2007). The latter may also come from value pluralism of experts (Rotmans and de Vries, 1997). A special category (with ontic and epistemic elements) is human reflexive uncertainty (d) originating from unknowns in human response to and anticipation of changes (Dessai and Hulme, 2004). Here, even when historical analysis suggests certain estimates by comparison and analogy, there is no guarantee that such an approach is valid for the time to come. All uncertainties together may result in total ignorance or deep uncertainty. Here, there is no agreement on the description of the system, the probability distribution of important drivers of the system or the value system used to rank alternatives (Lempert et al., 2004).

Other classifications of uncertainty can also be made: one refers to scale and distinguishes uncertainty in model parameters (1), uncertainty about model structure (2) and uncertainties that arise from (3) disagreements conceptual theories on an even larger scale.

As indicated, various methods have been introduced to deal with uncertainty in scenario development. In the field of greenhouse gas emission scenarios, focus was originally on "businessas-usual" emission trajectories, with simple variations for the main driving forces (e.g. Leggett et al., 1992). The two prominent approaches today, the alternative scenario approach and the fully probabilistic approach, can both be seen as an improvement to these early projections. The alternative scenario approach emphasizes the need for consistent assumptions and the handling of ignorance (cat. b2, b3, c, d), while the probability approach places the variations in the framework of a more structural assessment of plausible futures (cat. a, b1).

The IPCC-SRES scenarios, as most well-known application of the alternative scenario approach, map out a range of possible emission trajectories based on the wide variation in assumptions structured around four main storylines. Consistent with the basic premise of the approach, Nakicenovic and Swart (2000) indicate that it is not meaningful to assign probability estimates to these scenarios based on ignorance and the influence of societal choice (deep uncertainty). The SRES scenarios, however, formed the start of a lively debate. Schneider $(2001,2002)$ and Webster et al. (2002) argued that policy analysts and decision-makers need probability estimates to assess the risks of climate change impacts resulting from these scenarios; this is to decide how to respond to these risks. Even when probability estimates are subjective, in their view researchers (experts) are better equipped to make an assessment than the users (non-experts) of these scenarios. A counter argument from the SRES team (Grübler and Nakicenovic, 2001 ) that social systems (important in emission scenarios) are 
fundamentally different from natural science systems is dismissed by their critics: in both cases often probability estimates need to be made for systems that cannot be measured (Schneider, 2002). The absence of probability assignments in SRES also resulted in ambiguities in follow-up work such as in the probabilistic statements on temperature change made Wigley and Raper (2001), who decided to assign an equal likelihood to the scenarios.

Several studies have applied the contrasting probabilistic approach to emission scenarios (Manne and Richels, 1994; Nordhaus and Popp, 1997; Scott et al., 1999; Webster et al., 2002, 2003; Richels et al., 2004; Kouvaritakis and Panos, 2005; Pepper et al., 2005; Sweeney et al., 2006). An important critique formulated against this approach is that attempts to assign subjective probabilities in a situation of ignorance forms a dismissal of uncertainty in favor of spuriously constructed expert opinion (Grübler and Nakicenovic, 2001; Grübler et al., 2006). Moreover, it is also argued that while the fully probabilistic approach provides more (seemingly) readily useable information, the alternative scenario approach provokes creative thinking of decision-makers about possible futures and strategic choices. Finally, uncoupled sampling within distribution ranges of input parameters may result in inconsistent combinations. Clearly, the handling of uncertainty and the appropriateness of assigning subjective probabilities to scenarios is a matter of lively debate and an important, unresolved, challenge in the application of climate scenarios (Dessai et al., 2007; Groves and Lempert, 2007).

\subsection{Uncertainty approach used in this paper}

This paper applies the conditional probabilistic approach, as indicated in the Introduction, which is a combination of the scenario approach with formal uncertainty analysis. The approach attempts to combine the strength of the scenario approach in providing consistent descriptions of more fundamental uncertainties with the strengths of the formal uncertainty approach where appropriate. The rationale is that the reduction of the uncertainty space, with help of divergent storylines, will make uncertainties more suitable for a formal uncertainty method. For example, while it is difficult to assign meaningful probabilities to the future economic growth rate (as this depends on fundamentally uncertain factors such as trends in globalization), if one restricts the set of possible futures to only those in which globalization proceeds rapidly, the probability distribution of future economic growth rates is likely to narrow down. The approach was applied earlier to population scenarios by O'Neill (2004, 2005)

Obviously, the method proposed leaves some crucial questions open: (1) is it is possible to derive meaningful estimates of the parameter range conditional upon the scenario storyline, and (2) how can information derived by the method be used (also vis-àvis other methods of uncertainty analysis).

On the first point, one should realize that both the pure alternative scenario method and the full probabilistic approach need to make "arbitrary" estimates on how different variables evolve in the future (based on an interpretation of their past behavior). In the storyline approach this translates into a set of consistent assumptions of co-evolving parameters; in the fully probabilistic approach assumptions need to be made about reasonable sampling ranges. In that sense, the current approach adds by making these arbitrary choices somewhat more explicit. This allows for interpreting a "high growth storyline" by using a confined range of high economic growth assumptions and combining these with parameter ranges that are relatively plausible with such high growth, for instance, fast(er) technology change and a (more) rapid demographic transition. Admittedly, the degree of correlation between such ranges of qualitative storyline assumptions and model parameters are based on expert judgments_but as argued above: some mixture of expert elicitation and educated guesswork exists in any method that attempts to explore future developments.

As to their purpose, both the alternative scenario method and the derived method presented here are similar in that they do not intend to evaluate the likelihood of the underlying storylines. It still requires users to either accept that there are these fundamentally unknown elements or to make their own interpretation of the most appropriate storyline for their specific question (see Section 2.1 for the discussion between Schneider and SRES authors on the type of information that is needed (Grübler and Nakicenovic, 2001; Schneider, 2002)). The method, however, can be used to:

- Explore mission pathways-with ranges for different storylines and using statistical information for those parameters for which it is available.

- Allow for the analysis of robust strategies across different storylines-including the ranges that are associated with these storylines.

- Exploring the consistency of different storylines-and the role of individual parameter uncertainty within each storyline.

- As compared to the unconditional probabilistic approach: explore the impacts of linkages among different parameters (which are usually ignored) and making the sampling of parameters ranges more explicit.

The current method is not meant to replace the existing methods as they are used for different purposes and respond to different types of uncertainty. It rather provides a complementary tool to deal with large and hard-to-quantify uncertainties. We come back to the limitations and use of the method in the Discussion section.

In terms of the actual application, we based our analysis on the IPCC-SRES scenarios (Fig. 1) (Nakicenovic and Swart, 2000). These scenarios are described in Section 2.3. The scenarios and storylines considered in this paper all represent so-called baseline scenarios; i.e. we assume no climate policy. Uncertainties with respect to technologies such as carbon capture and sequestration (CCS), which are only relevant in a world that includes climate policies, are therefore not included in the analysis. We also consider only $\mathrm{CO}_{2}$ emissions from energy use. Our conditional probabilistic analysis consisted of the following four steps:

1. Identification of parameters subject to uncertainty analysis.

2. Assessment of the conditional probability ranges associated with these parameters.

3. Use of Monte-Carlo sampling to calculate uncertainty results and TIMER model runs.

4. Identification of ranges for model outcomes and of determinants adding to model uncertainty.

For step 1, we used the results of an earlier uncertainty analysis on the TIMER energy model that was based on the NUSAP method (van der Sluijs et al., 2002). This analysis used several techniques to identify elements of uncertainty in TIMER, including a formal sensitivity analysis, a 2-day expert elicitation workshop, and model comparison and interview techniques with different model developers. Based on this study, we identified the most relevant model parameters to include in a formal uncertainty analysis (either based on relevance or sensitivity). Step 2 was to quantify the probability functions of those model parameters conditional to the scenario storyline of the model (see Section 2.5). 


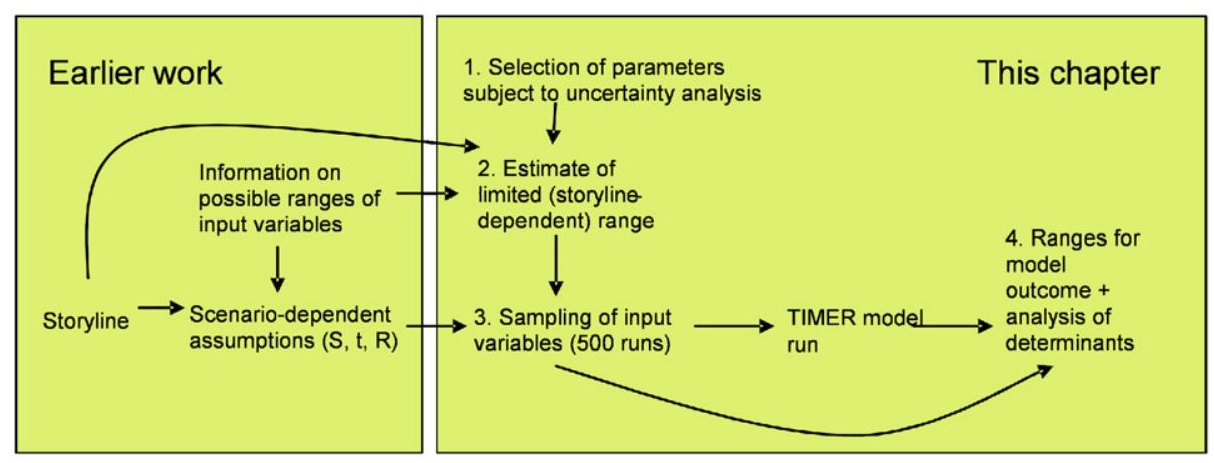

Fig. 1. Overview of the analysis $(S=$ scenario, $t=$ time; $R=$ region $)$

Next (step 3), we applied Monte-Carlo sampling of input data for 750 model runs and estimated (step 4) the probability range for outcome parameters, and the contribution of the uncertainty ranges assigned to different parameters (see Section 2.6).

\subsection{The TIMER energy model}

In this analysis we used the TIMER 2 energy model (Van Vuuren, 2007). TIMER is a system-dynamics simulation model at an intermediate level of aggregation: 17 world regions, five energy-demand sectors and around 10 different energy carriers. TIMER is a simulation model: it simulates year-to-year investment decisions based on specific rules about investment behavior, fuel substitution and technology. The time horizon in the present analysis is the period from 2000 to 2100 , while model calibration is performed on the basis of historical data for the 1971-2000 period.

In the model, energy demand is calculated on the basis of changes in sectoral value-added and GDP, population, income elasticities, autonomous-energy efficiency improvement (AEEI) and price-induced efficiency improvement (PIEEI) (see Fig. 2). Market shares of various energy carriers in each sector are determined by means of multi-nomial logit equations, taking into account changes in price and/or subscribed fuel preferences. Demand for electricity and hydrogen are forwarded to submodels that simulate investments in various technological options (both fossil-fuel based, bio-energy based and non-fuel-based technologies) to produce these final energy carriers. The decisions on investments and fuel use are derived from the relative (perceived) costs of each option, according to a multi-nomial logit formulation. Demand for primary energy carriers (fossil fuels and bioenergy) are finally fed into different production models that simulate their production and trade. The costs of energy carriers result from an interplay between depletion and learning dynamics. Depletion leads to increasing production costs, as a function of cumulative production of fossil fuels or of the ratio between actual and maximum potential in the case of renewables. Learning-by-doing leads to a decrease in production costs.

\subsection{Storylines of the IPCC-SRES scenarios}

Nakicenovic and Swart (2000) provide a detailed description of the SRES scenarios, organized around the two major uncertainties in the direction that the world could evolve. These are globalization versus regionalization, and economic orientation versus orientation towards social development and environmental protection (resulting in four scenario families A1, A2, B1 and B2). Other dimensions are considered to be implicitly or explicitly related to these two dimensions, for instance, technology and governance.

The storyline of the A1 scenario is based on an assumed continuation of globalization trends and a focus on market processes and economic objectives. Within the logic of the storyline, economic growth is assumed to be high. As this could spur on the demographic transition, population growth in turn is low. In terms of the energy system, the scenario is characterized by rapid technology development but also by energy-intensive lifestyles. Within the A1 storyline, there are three variants based on the emphasis in technology development: (1) balanced (A1b), (2) fossil-intensive (A1FI) and (3) focused on renewable technology (A1T). The A2 storyline, in contrast, emphasizes regional (energy) security and cultural identity. Here, it is assumed that trade protectionism and barriers between world regions will slow down technical innovations and economic growth, which will, in turn, tend to slow down the demographic transition in lowincome regions. The B1 storyline describes a convergent world with emphasis on global solutions to environmental and social sustainability, including concerted efforts towards reduction of economic inequity, and less energy- and material-intensive products and lifestyles ("dematerialization"). Finally, on the basis of its position with respect to the major uncertainties, the B2 storyline emphasizes regional sustainable development. However, for practical reasons this scenario is mainly implemented as a combination of medium assumptions for several trends.

Although the SRES scenarios as originally implemented are still broadly consistent with the literature, new insights have emerged for some parameters (van Vuuren and O'Neill, 2006). For instance, current expectations for population and economic growth for lowincome regions are now generally lower than that assumed in SRES. Against this background, a set of updated scenarios was recently developed using the Integrated Model to Assess the Global Environment (IMAGE), the integrated assessment modeling framework of which TIMER forms the energy model (van Vuuren et al., 2007) (see Fig. 3). These scenarios form the starting point of the analysis presented here. We have decided to comply with the tradition of sometimes placing the B2 storyline in the middle of the three other, more explicitly focused, storylines. We assume that the alternative variants in the A1 world (A1B, A1FI and $\mathrm{A} 1 \mathrm{~T}$ ) can be generated in the analysis by varying technology parameters on the basis of statistical uncertainty analysis in the A1 storyline-and thus need not to be specified explicitly.

\subsection{Parameter values and their ranges}

The most sensitive parameters identified in the quantitative and qualitative uncertainty analysis for the TIMER model reported by van der Sluijs et al. (2002) has been used as starting point for 


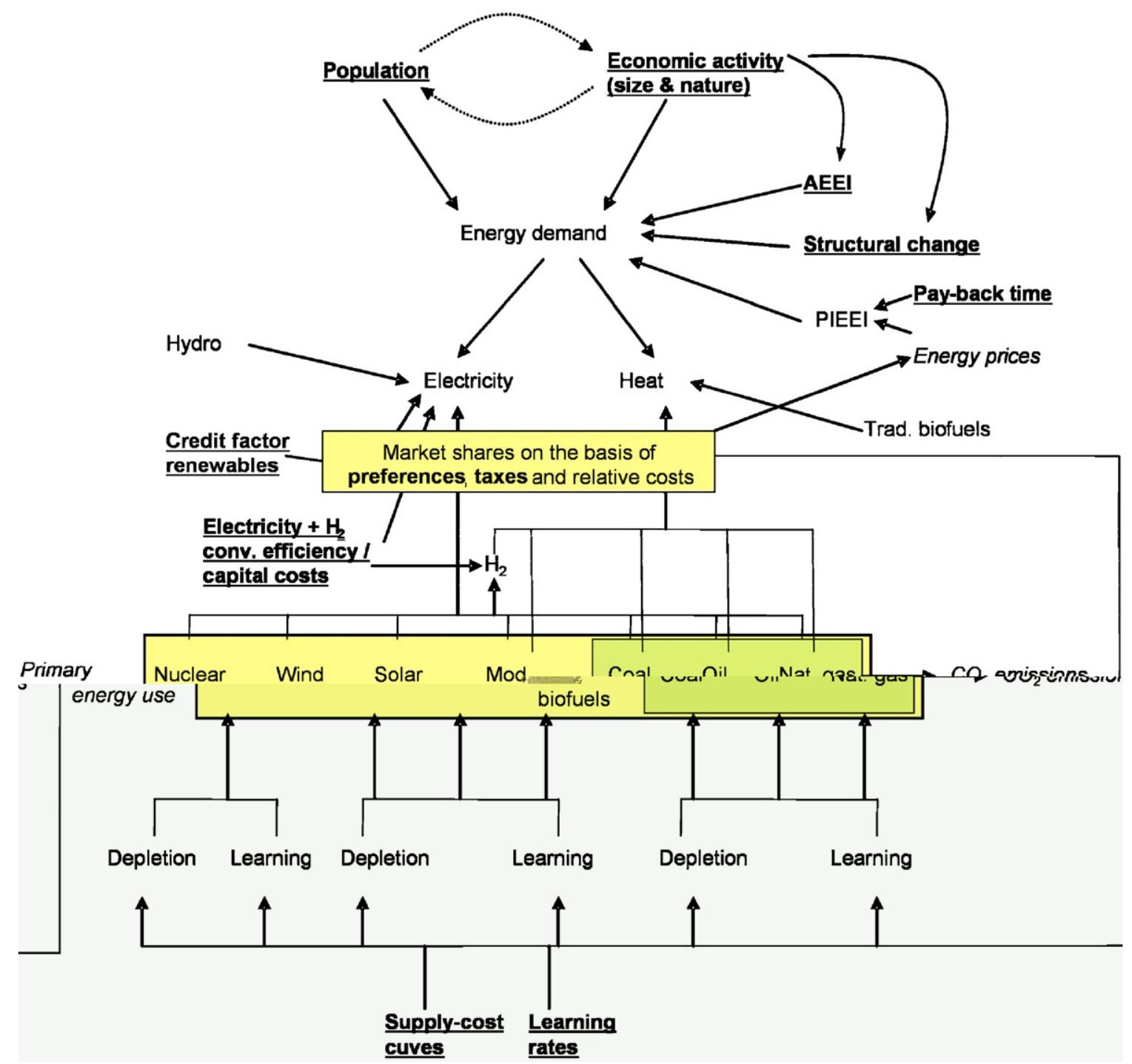

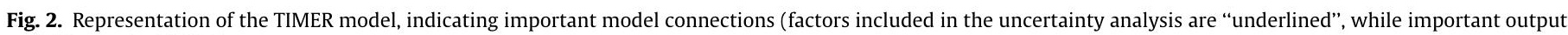
variables are in "italic").

selecting uncertainty parameters considered in this study. ${ }^{2}$ Moreover, their expert elicitation was used in the specification of useful parameter ranges. The list of input parameters is given in Table 1 (see also Fig. 2). Assumptions for our uncertainty analysis were made on a global scale, unless additional information was available that allowed regional specification. Webster and Cho (2006) recently showed that using a more limited correlation of regional growth rates (i.e. correlation only bound by the empirically observed level) led to a considerably reduced range of outcomes for $\mathrm{CO}_{2}$ emissions compared to the original work of Webster et al. (2002) (which assumed full regional correlation). As a result, also the full correlation assumed here may result in broader ranges in output variables than in the situation where no perfect correlation has been assumed.

For each parameter, we use as mean value the assumptions of the recent TIMER elaboration of the IPCC-SRES scenarios (van Vuuren et al., 2007). The sampling ranges around these means have, as far as possible, been based on ranges indicated in

\footnotetext{
${ }^{2}$ Some parameters (technology assumptions for $\mathrm{H}_{2}$, wind/PV resources and capacity credit) were added later in association with model additions made more recently.
}

the literature, such as historical fluctuations or explicit statements on their distribution. In the Appendix A, we explicitly describe the sampling ranges of the different parameters and the reasoning behind the choices made. In each case, we believe that the range chosen does adequately represent the storyline of the scenarioalso in light of the purpose of the method (see Section 2.2). As indicated in Table 1, for most parameters, the sampling range is set the same for all scenarios and regions. Population and economic growth form an exception, as here the sampling ranges are also scenario- and region dependent.

It should be noted that estimating the sampling range is complex. If ranges (or even probability distribution functions (pdfs)) are found in the literature, these often refer to what are meant as unconstrained situations (i.e. not depending on certain storylines). This introduces an element of arbitrariness as these estimates need to be interpreted in the context of our storylines (see also Section 2.2). Although expert elicitation would be a preferred instrument to do this, for the sake of simplicity and time, the ranges here were only partly based on expert elicitation (van der Sluijs et al., 2002) and partly by interpretation of available literature by the authors of this paper. The overall scheme used in this interpretation process is shown in Fig. 4. As an example an unconditional range is shown on the left-hand side 

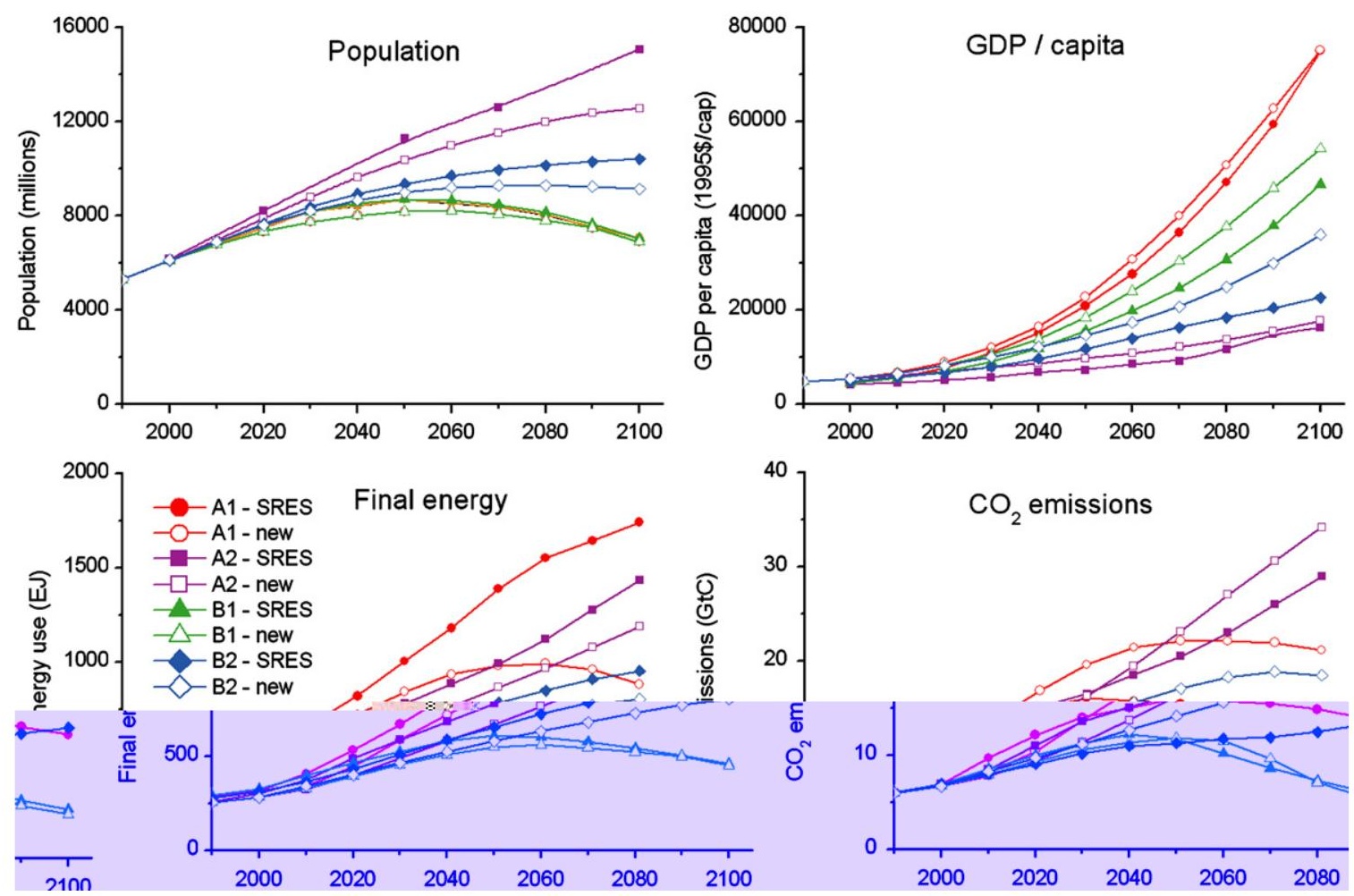

Fig. 3. Driving forces and fossil fuel $\mathrm{CO}_{2}$ emissions in the IMAGE 2.3 SRES scenarios compared to the IPCC-SRES Marker scenarios (Nakicenovic and Swart, 2000) (see also www.ipcc.ch)

Table 1

Input parameters included in uncertainty analysis

\begin{tabular}{|c|c|c|c|}
\hline Parameter category & Parameter & Central value & $\begin{array}{l}\text { Sampling range around } \\
\text { central value }\end{array}$ \\
\hline Driving forces & $\begin{array}{l}\text { Population } \\
\text { GDP } \\
\text { Size of industry sector }\end{array}$ & $\begin{array}{l}\text { Scen, Reg } \\
\text { Scen, Reg } \\
\text { Scen, Reg }\end{array}$ & $\begin{array}{l}\text { Scen, Reg } \\
\text { Scen, Reg } \\
\text { Indep. }\end{array}$ \\
\hline Energy demand & $\begin{array}{l}\text { AEEI } \\
\text { Pay-back time } \\
\text { Structural change }\end{array}$ & $\begin{array}{l}\text { Scen, Reg } \\
\text { Scen, Reg } \\
\text { Scen, Reg }\end{array}$ & $\begin{array}{l}\text { Indep. } \\
\text { Indep. } \\
\text { Indep. }\end{array}$ \\
\hline Technology change & $\begin{array}{l}\text { Fossil fuels } \\
\text { Renewables (electric power) } \\
\text { Nuclear power } \\
\text { Bio-energy } \\
\text { Energy demand } \\
\text { Hydrogen technologies } \\
\text { Thermal power plants }\end{array}$ & $\begin{array}{l}\text { Scen, Reg } \\
\text { Scen, Reg } \\
\text { Scen, Reg } \\
\text { Scen, Reg } \\
\text { Scen, Reg } \\
\text { Scen, Reg } \\
\text { Scen, Reg }\end{array}$ & $\begin{array}{l}\text { Indep. } \\
\text { Indep. } \\
\text { Indep. } \\
\text { Indep. } \\
\text { Indep. } \\
\text { Indep. } \\
\text { Indep. }\end{array}$ \\
\hline Resources & $\begin{array}{l}\text { Oil resources } \\
\text { Gas resources } \\
\text { Coal resources } \\
\text { Wind resource } \\
\text { Biomass resource } \\
\text { PV resource }\end{array}$ & $\begin{array}{l}\text { Reg } \\
\text { Reg } \\
\text { Reg } \\
\text { Reg } \\
\text { Scen, Reg } \\
\text { Reg }\end{array}$ & $\begin{array}{l}\text { Indep. } \\
\text { Indep. } \\
\text { Indep. } \\
\text { Indep. } \\
\text { Indep. } \\
\text { Indep. }\end{array}$ \\
\hline Other & $\begin{array}{l}\text { Fuel preferences } \\
\text { Credit factor for renewables } \\
\text { Taxes } \\
\text { Short-term price uncertainty oil and gas }\end{array}$ & $\begin{array}{l}\text { Scen, Reg } \\
\text { Reg } \\
\text { Scen, Reg } \\
\text { Reg }\end{array}$ & $\begin{array}{l}\text { Indep. } \\
\text { Indep. } \\
\text { Indep. } \\
\text { Indep. }\end{array}$ \\
\hline
\end{tabular}

Scen: indicates that either the central value or the sampling range around this value is scenario-dependent.

Reg: indicates that either the central parameter value or the sampling range around this value is region-dependent.

Indep.: indicates that the sampling range is scenario- and region-independent (thus a constant sampling range).

for a selected input variable as found in the literature (e.g. a 95\% interval). For those parameters for which pdfs were available (progress ratios, population), the shape was mostly comparable to a normal distribution. On this basis, we have (again for the sake of simplicity) assumed all parameters to be normally distributed. Next, storyline descriptions were used to choose a specific range within the unconditional pdf for each scenario. As most storyline statements are described as "high", "low" or "medium", a 
a

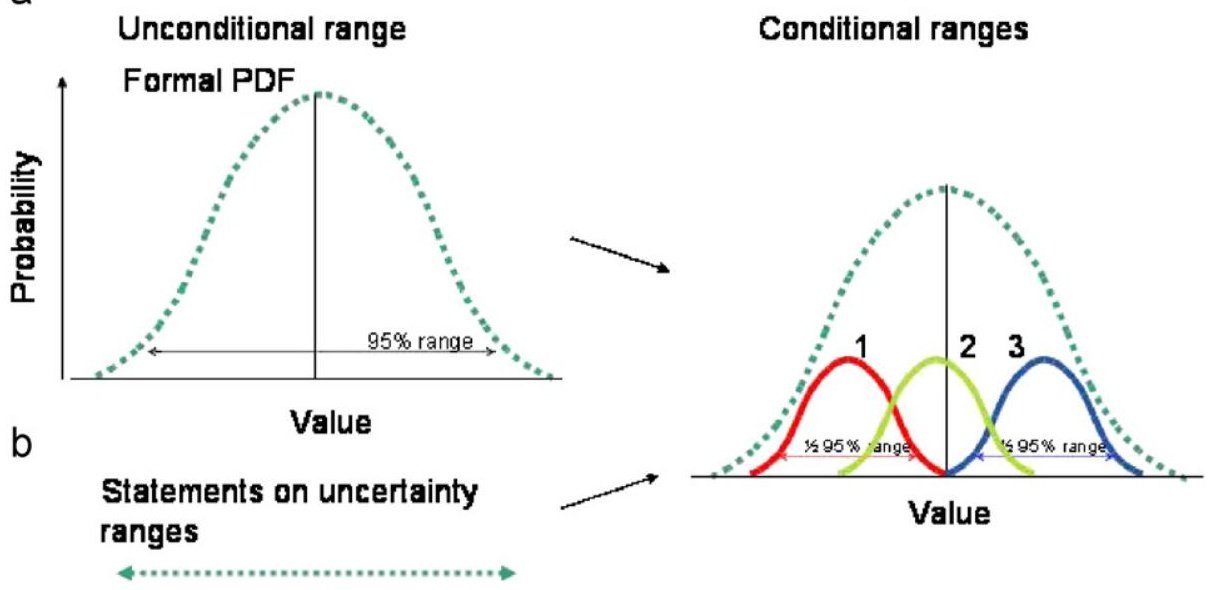

Fig. 4. Scheme used in interpretation process showing derivation of conditional ranges (if those were not available).

standard interpretation was made. We assumed that these statements generally refer to values above, below or near the median value, respectively, thus assigning a corresponding half of unconditional 95\% interval to each scenario (see Fig. 4). This implies that, unless more specific information had been available, our conditional distribution was characterized by main value, based on the existing scenario implementation of van Vuuren et al. (2007), with an uncertainty range equal to half the 'unconditional range'.

Clearly, pdfs of different parameters are not unrelated. Relationships may exist in the form of interactions outside the scope of the model or in the form of the scenario storyline. For instance, the A1 storyline emphasizes that its high economic growth is likely to spur on the demographic transition leading to low population growth. Or, in another example, the relatively slow rate of technological change in the A2 scenario is considered to be in line with the low economic growth rate, which, in turn, is an assumed consequence of trade protectionism. As our approach captures the original implementation of the scenarios and only samples around these "median" values, the existing qualitative relationships between model parameters are arguably preserved.

\subsection{Parameter sampling and analysis}

In order to limit computational load we use the Latin hypercube sampling (LHS) technique. LHS can be used in combination with linear regression to quantify the sensitivity and uncertainty contributions of the input parameters to the model outputs (Saltelli et al., 2000, 2004). On this basis, 750 runs are made for each scenario, sampling values for each of the 26 input values $\left(X_{i}\right)$. In the analysis of the output data, the values for each output variable $Y$ (e.g. $\mathrm{CO}_{2}$ emissions) are approximated by a linear function of the inputs $X_{i}$, expressed by

$Y=\beta_{0}+\beta_{1} X_{1}+\beta_{2} X_{2}+\cdots+\beta_{n} X_{n}+e$

where $\beta_{i}$ is the so-called ordinary regression coefficient and $e$ the error of the approximation. The quality of the regression model is expressed by the coefficient of determination $\left(R^{2}\right)$ representing the amount of variation in $Y$ explained by $Y-e$. Next, we use the standardized regression coefficient (SRC), which is a relative sensitivity measure obtained by rescaling the regression equation on the basis of the standard deviations $\sigma_{Y}$ and $\sigma_{X_{i}}$ :

$S R C=\beta_{i} \frac{\sigma_{X_{i}}}{\sigma_{Y}}$
SRCs can take values between -1 and 1 . SRC is the relative change $\Delta y / \sigma_{y}$ of $Y$ due to the relative change $\Delta x_{i} / \sigma_{X_{i}}$ of the parameter $X_{i}$ considered (both with respect to their standard deviation $\sigma$ ). Hence, $S R C$ is independent of the units, scale and size of the parameters. Its value is indicative of the contribution of the uncertainty in $X_{i}$ in the uncertainty of $Y$. The sum of squares of $S R C$ values of all parameters equals the coefficient of determination, which for a perfect fit equals 1 . An absolute SRC value above 0.2 (contributing more than $4 \%$ ) is indicative of a strong relationship, provided that its contribution is also significant. Testing whether $S R C$ is significant is done with Student's t-statistic (Saltelli et al., 2000). The $S R C$ is significantly different from zero if the absolute value of Student's $t$-statistic exceeds 2 . It is important to note here that any conclusions drawn from the regression model are only valid if the $R^{2}$ is indeed close to 1 , i.e. the regression model is indeed a fair approximation. Commonly, a value above 0.8 is considered acceptable. Furthermore, any statements about the $S R C$ s are made under the assumption that the input parameters are uncorrelated.

\section{Results}

We use the so-called Kaya identity as a framework for discussion of our results. The Kaya is presented below:

$\mathrm{CO}_{2}$ emis $=$ Pop $* \frac{G D P}{\text { Pop }} * \frac{\text { EnergyCons }}{G D P} * \frac{\mathrm{CO}_{2} \text { emis }}{\text { EnergyCons }}$

where $\mathrm{CO}_{2}$ emis stands for emissions of $\mathrm{CO}_{2}$, Pop for population size, GDP for economic output, and EnergyCons for primary energy consumption. The factor EnergyCons/GDP (energy intensity) is a function of energy efficiency improvement and changes in the structure of the economy. The factor $\mathrm{CO}_{2}$ emis/EnergyCons (carbon factor) is a function of the mix of primary energy carriers. While Section 3.2 focuses on developments in energy intensity and in the carbon factor, Section 3.3 looks into changes in the mix of primary energy carriers.

Table 2 summarizes the information found on the SRC (average value over $2000-2100$ period). Results of Table 2 are included in the discussion of the results further on in this paper.

\subsection{Trends in $\mathrm{CO}_{2}$ emissions}

The $\mathrm{CO}_{2}$ emissions calculated by the TIMER model on the basis of these scenarios covers a broad interval (4-40 GtC in 2100) (Fig. 5). The emission trajectories are not surprising: for each 
Table 2

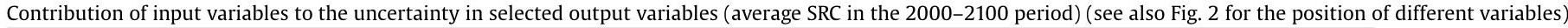

Input parameters

$$
\text { Section } 3.1 \text { Section } 3.2
$$

Output parameters

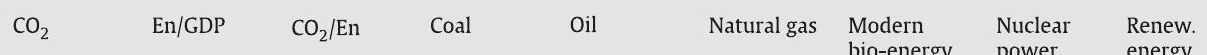

Driving forces

Population

GDP

\begin{tabular}{|c|c|c|c|c|c|c|c|c|c|c|c|c|c|c|c|c|c|}
\hline 0.29 & 0.26 & 0.01 & 0.01 & 0.04 & 0.11 & 0.19 & 0.16 & 0.18 & 0.22 & 0.17 & 0.22 & 0.11 & 0.16 & 0.2 & 0.18 & 0.19 & 0.23 \\
\hline 0.81 & 0.37 & 0.06 & 0.04 & 0.22 & 0.05 & 0.66 & 0.3 & 0.65 & 0.25 & 0.55 & 0.2 & 0.32 & 0.16 & 0.62 & 0.28 & 0.41 & 0.19 \\
\hline 0.58 & 0.63 & 0.74 & 0.66 & 0.11 & 0.14 & 0.19 & 0.21 & 0.4 & 0.48 & 0.2 & 0.26 & 0.16 & 0.14 & 0.29 & 0.31 & 0.15 & 0.13 \\
\hline 0.5 & 0.69 & 0.59 & 0.66 & 0.21 & 0.2 & 0.34 & 0.35 & 0.43 & 0.45 & 0.28 & 0.25 & 0.19 & 0.2 & 0.37 & 0.49 & 0.23 & 0.19 \\
\hline 0.07 & 0.07 & 0.1 & 0.11 & 0.02 & 0.02 & 0.04 & 0.03 & 0.05 & 0.08 & 0.03 & 0.04 & 0.03 & 0.03 & 0.02 & 0.02 & 0.03 & 0.04 \\
\hline
\end{tabular}

Size of industry sector

scenario the median values follow a pattern consistent with the marker IPCC scenarios. In the case of A1, rapid economic growth results first in a sharp increase in emissions which levels off after 2050 , mainly as a result of a stabilizing population. Under A2, emissions grow slowly first (as a result of slow economic growth), but continue to grow in the second half of the century, driven by further population growth and an increasing share of coal use (see further on). The B2 scenario shows an intermediate pattern 


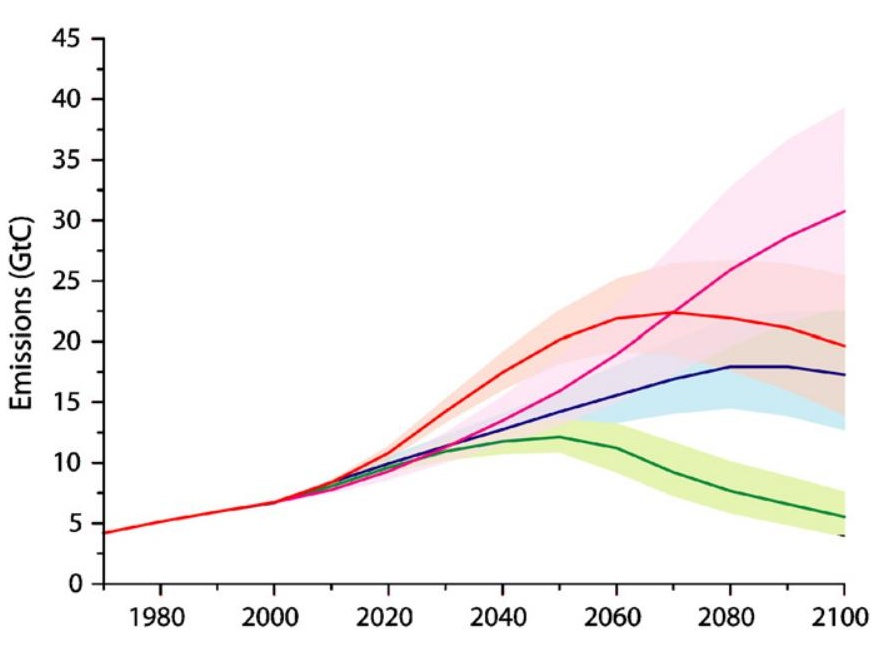

Fig. 5. $\mathrm{CO}_{2}$ emissions as a function of time.

throughout the century, while the B1 scenario follows a pathway that clearly differentiates from other scenarios, peaking already around 2050. Here, the assumed (normative) "pro-active" assumption with respect to fuel choice and the fast technology change lead to very different results than other scenarios after 2050.

Of importance here are not so much the median values, but the formalized uncertainty ranges. Fig. 5 shows a relatively strong overlap between the $95 \%$ interval ranges of the A1, B2 and A2 storylines, and of B1 up to 2040. The A1 scenario range, however, lies above the range of other scenarios as a result of high economic growth assumptions, but results are more widespread in the second half of the century, overlapping almost completely with the B2 range (around 15-25 GtC). The 2000-2100 cumulative emissions (Fig. 6) range from an annual average of $800-1200 \mathrm{GtC}$ for B1 to 1200-2500 GtC for the other scenarios. The "mediumassumption" scenario B2 range overlaps with the low-end range of "high growth" A1 and "fragmentation scenario" A2. The A2 shows the widest range of all three scenarios, extending both on the lower and upper sides beyond the $A 1$ range. The peaks in the pdfs for the A1, A2 and B2 scenarios are in close proximity to each other, with an average annual value of 1500-2000 GtC.

Table 2 shows that the most important determinants of global carbon emissions are the input factors that determine energy demand: income, population, efficiency improvement and structural change. Other factors that play a role are uncertainty in fuel preferences, technology improvement rates for renewables and energy demand and oil resources. Interestingly, other factors are important for different storylines. For instance, population is relatively important in A2, autonomous efficiency improvement in A1 and fuel preferences in A1 and B1. It is very interesting to note that these observations are consistent with the original storyline-and confirm added value of the conditional approach: different parameter uncertainties are important under different storylines.

\subsection{Energy intensity and the carbon factor}

For energy intensity (Fig. 7), all scenarios show a distinct improvement: most progress occurs in B1 and the least improvement in $A 2$. The uncertainty range around the development path of B1, A1 and B2 partly overlap. The development pattern occurring in the A2 scenario is clearly distinct (slow) as a result of the relatively slow development of GDP and unfavorable technology assumptions. The uncertainties determining the

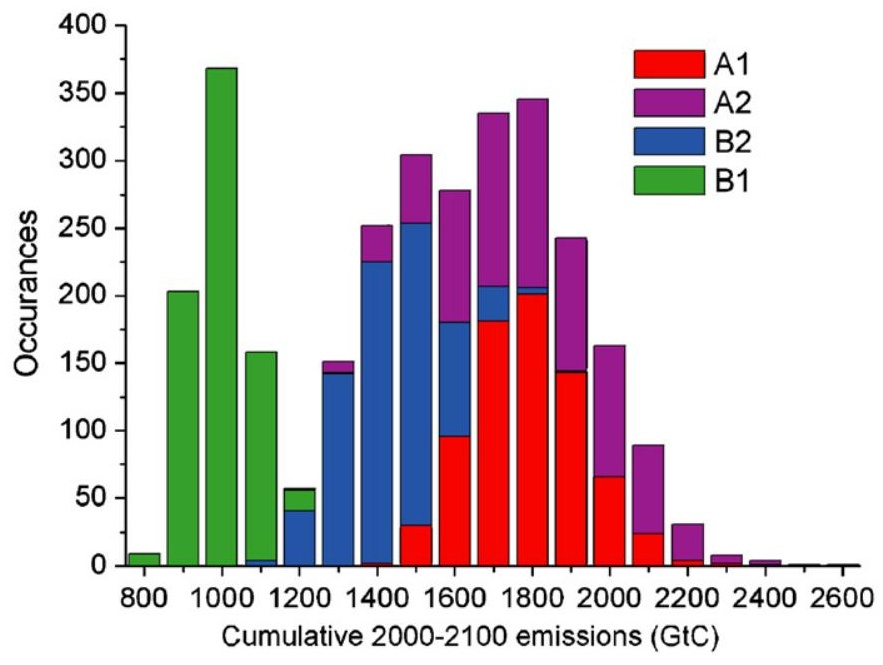

Fig. 6. Frequency distribution of cumulative emissions 2000-2100.

energy intensity improvement (see Table 2) are GDP, AEEI, structural change (both between and within sectors), the oil resource and fuel preferences. Short-term uncertainty in energy prices also plays a role. The influence of the first three factors can be readily understood from assumed model relationships (GDP drives AEEI and structural change), while other factors operate via PIEEI.

A very wide range of results is found for development of the carbon factor $\left(\mathrm{CO}_{2}\right.$ emissions per unit of energy) strongly related to the storylines. In contrast to energy intensity, the carbon factor has been nearly constant over the last 30 years (indicating a relatively constant energy mix). This trend is continued in the "medium" B2 range-although by the end of the century, depletion of fossil fuels results in a distinct drop. In the $\mathrm{A} 2$ range, the opposite happens as a result of a move towards coal (see further). The A1 range lies somewhat lower due to optimistic technology assumptions (important for the penetration of non-fossil-based technologies). Finally, the carbon factor for B1 rapidly declines-driven by the focus on renewable resources. The uncertainty ranges are larger for B2 and A1 than in the other two scenarios resulting from the fact that their storyline is less binding for fuel choice (B1 focuses on renewable sources, while $A 2$ is forced into coal due to trade restrictions). In addition to the factors that impact energy demand, the uncertainties in fuel preferences and several resource and technology parameters contribute to the ranges found for the carbon factor. Again, the contribution of the different factors depends on the storyline. Uncertainty in GDP growth is relatively important for the uncertainty in energy intensity in the A1 scenario; while the uncertainty in structural change is relatively important in B1 and B2. For the carbon factor uncertainty, population and gas resources stand out in A2 (both influencing depletion dynamics in this scenario) and technology development for renewables in B1.

\subsection{Fuel mix}

Fig. 8 shows the global consumption of coal, oil, natural gas and renewables in each of the scenarios. As can be seen in Fig. 2, these fuels are substitutes. Three factors play a major role in substitution: fuel preferences, technology change and depletion.

The availability of extractable fossil fuel, in particular oil, makes resources a current subject of debate (Witze, 2007). Some believe that the world has already reached a maximum rate at which oil can be produced and further depletion will force consumption to decline (the so-called peak-oil hypothesis). 

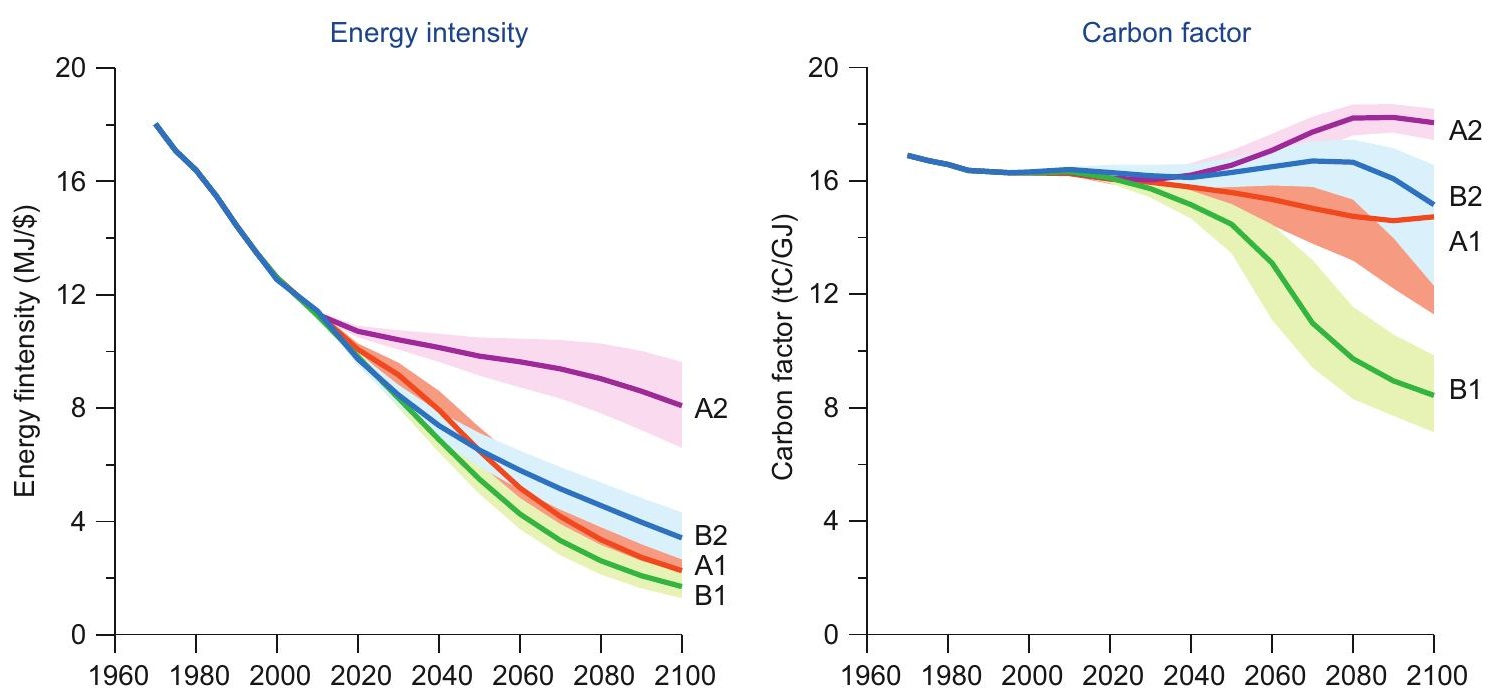

Fig. 7. Development of the Kaya indicators.

Others, however, claim that there will not be real limits on oil production for the next 30 years. Here, we have based the uncertainty ranges for conventional resources on the probabilistic statements of USGS (as summarized in Mulders et al., 2006). On the low side, the USGS estimates coincide reasonably with those of peak-oil proponents (Laherre and Cambell, 1999; Deffeyes, 2006). On the high side, the USGS estimates are consistent with claims that there will be abundant oil resources available in the next decades (Witze, 2007). For unconventional fossil resources, the situation is more complicated as probabilistic resource estimates have not been established and estimates vary from hardly any extractable reserves to nearly unlimited supplies. Here, we vary unconventional fossil fuel estimates over a wide range, but based on the large estimates of these resources, their availability continues to dominate supply as indicated in Fig. 9. ${ }^{3}$

In our results for oil a clear peak in consumption levels occurs in about half of the scenarios. However, such a peak occurs in different periods, at different levels and for different reasons. In fact, even for high resource estimates, oil use is likely to peak as a result of saturating energy demand (driven, for example, by a stabilizing world population) in combination with slowly rising prices. Low-resource assumptions in combination with competitive alternatives show a peak in oil use before 2040. In our calculations, the extreme form of the peak-oil theory (an oil peak before 2010) cannot be reproduced given: (1) assumed inertia, (2) availability of large unconventional resources and (3) the fact that no explicit model relationship exists between the extraction rate and the degree of depletion (part of the peak-oil hypothesis). Table 2 shows that the range of oil consumption pathways is determined by energy demand, the size of the oil resource and the technology factors for fossil fuel production. In addition, the assumed potential of oil's main competitor, bio-energy plays a role (both resource size and technology development).

Fig. 9 compares the long-term supply-cost curves under the low, medium and high resource estimates. Sampling is done in between these three extremes (in time, the curve changes by moving to the left along the $x$-axis as a result of technology

\footnotetext{
${ }^{3}$ In this study, we applied a factor 2 variation, upwards and downwards, in unconventional resources. This range, however, is not wide enough to fully capture the very low reserve estimates of oil-peak proponents, nor does it capture a deliberate choice to refrain from developing these resources for environmental reasons.
}

development). In the figure, resource availability is compared to 2050 and 2100 cumulative consumption levels. As shown, under the medium assumptions, conventional oil is more-or-less depleted around 2050. However, the large amounts of unconventional resources are still available for exploitation. If supply is more limited, by 2050 also the most accessible unconventional resources are likely to be depleted, while at the other end of the range, high estimates imply that even by 2050, conventional resources have only been exploited by about two-thirds. In 2100, cumulative consumption levels vary from 3000-5000 billion bbls, in which the majority of consumption comprises non-conventional resources under each set of assumptions. As such scenarios imply a transition to unconventional oil resources, the uncertainty in production costs, the associated impacts on the environment, and the gross greenhouse gas emissions deserve further attention.

Uncertainty in natural gas use is determined (Table 2), apart from demand factors, by gas resources, short-term fuel price uncertainty, technology development for fossil fuels, oil resources (as substitute) and fuel preferences. Fig. 9 shows that at similar cost levels, more natural gas than oil is available. Correspondingly, natural gas use grows more rapidly than oil use (assuming no constraints on infrastructure investment) but still peaks after 2040-2060. The main reason is that resource depletion results in higher natural gas prices and, given the flexibility of fuel choice in the power sector, leads to relatively easy substitution away from natural gas.

For coal use, a distinct difference is found between the B1 scenario and the other three scenarios (Fig. 8) caused by the assumed preferences in B1 for clean fuels. In all other scenarios, coal consumption in the absence of climate policy is likely to increase. Coal use in 2100 ranges from $30 \mathrm{EJ}$ to a staggering $1000 \mathrm{EJ}$. On the high side, the A2 scenario dominates the overall range. The uncertainty in coal use is determined by similar factors to those for natural gas use, although here too, the uncertainty in renewables in the power sector plays an important role.

Finally, the trajectories for other energy carriers (renewables and nuclear) show a rapid expansion in all cases. The highest values are found for the B1 and A1 scenario (in B1, rapid technology development and a preference for clean fuels are major drivers; in A1, a major driver is rapid technology development in combination with high energy demand). As the A1 range is wider than the B1 range, the highest values are, in fact, found 


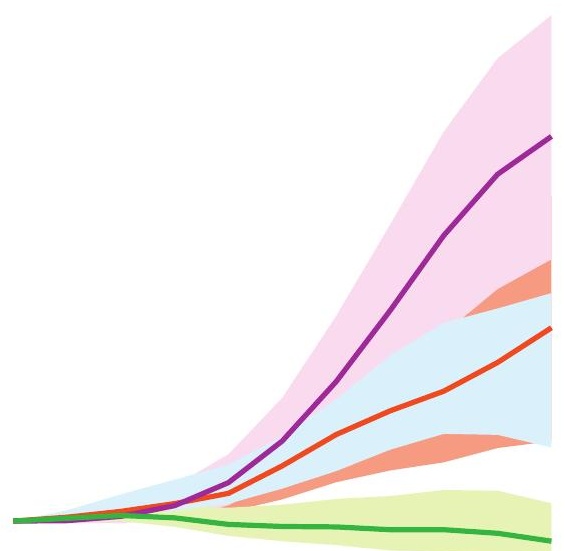

under the A1 storyline. The lowest values are found under the A2 and $\mathrm{B} 2$ scenarios.

The trends as discussed here are also depicted in Fig. 10 which shows shares in total consumption (note that scenarios have very different overall consumption levels.) In the first decades, all scenarios move in the direction of increasing shares of oil/ gas-followed by a decrease as a result of increasing prices (thus reducing competitiveness with other forms of energy). In the B1 
scenario, the response is to go in the direction of an energy system consisting of primarily renewable energy. The A2 scenario responds differently to increasing oil/gas prices by moving in the direction of coal. The uncertainty range surrounding this scenario is smaller. The other 2 scenarios follow a more intermediate trajectory.

Underlying the fuel choice in the model are the energy prices and production costs. As indicated in Fig. 2, production costs are a function of depletion and learning-by-doing; both are driven by cumulative production. These costs are shown for fossil fuels in Table 3. Interestingly, the differences between the scenarios are rather small-given the feedbacks in the model: scenarios with relatively abundant resources or rapid technology development lead to high exploitation rates and thus, indirectly, to higher prices. For oil, the scenarios indicate a 2-3.5-fold increase in oil prices across the century. For gas, an even higher increase is found. In contrast, coal prices increase only modestly (certainly in absolute numbers).

\section{Discussion and comparison to other approaches}

In the Introduction, we have already indicated that uncertainty can be classified in different ways. Obviously, the source or type of uncertainty has important consequences for the way it needs to be managed in scenarios. Different methods were applied in the literature to deal with uncertainty. In addition to alternative scenarios and the full probabilistic approach, also other methods have been applied such as model comparison (e.g. Weyant et al.,

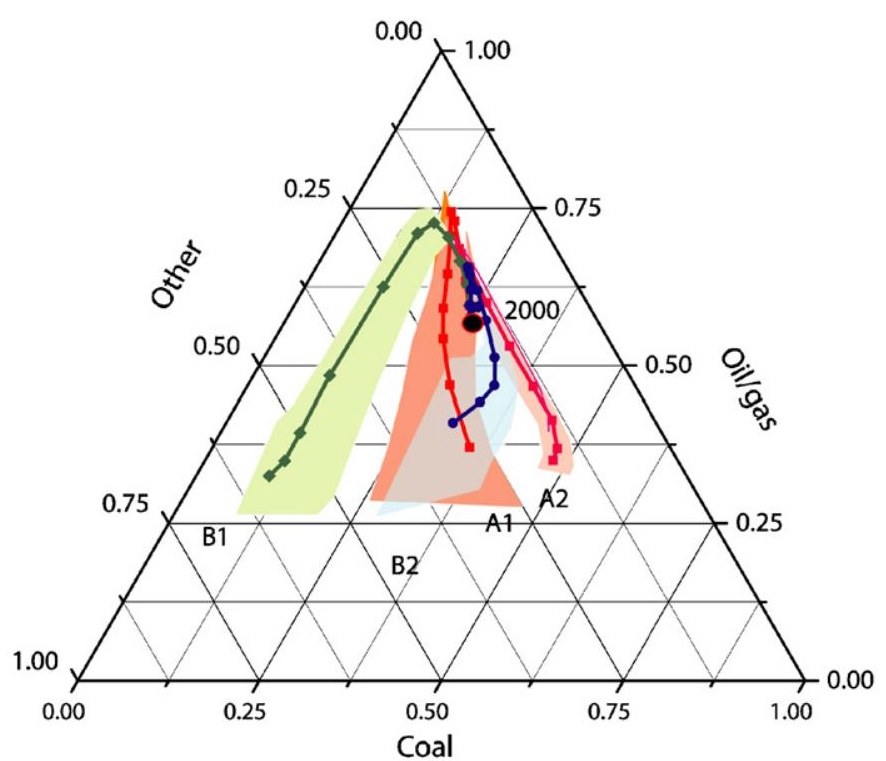

Fig. 10. Primary energy expressed in the contribution of three main categories: coal, oil/gas and other (bio-energy and non fossil-based electric power). The corners of the triangle indicate $100 \%$ other (left-bottom), $100 \%$ coal (right-bottom) and $100 \%$ oil/gas (top).
2006) and the NUSAP method (van der Sluijs et al., 2002). Each of the uncertainty methods relate in a different way to the sources of errors indicated above. With respect to sources of uncertainty, formal probability analysis, in particular, addresses ontic uncertainty and statistical representations of epistemic uncertainty (a-b1; see Section 2) by expressing uncertainty ranges in pdf of input variables. In terms of scale, the uncertainty addressed by this method occurs mostly at the level of parameters (1). The alternative scenario method, in contrast, addresses epistemic or human reflexive uncertainty (b2, c, d), in particular, by varying values of input parameters across the scenarios. In terms of scale, the scenario method focuses on the level of parameters (1), but by adding storylines outside the model on more conceptual issues (3). Model comparison as a method to deal with uncertainty is particularly relevant for uncertainty originating from value pluralism and ignorance on model relationships (c, 2). By comparing different models some model-based biases can be made explicit (although collective bias will not be detected).

Earlier scenario studies can be classified on the basis of the methods discussed above (Fig. 11). The studies of Webster et al. (2002), Sweeney et al. (2006) and Kouvaritakis and Panos (2005) can be interpreted as applications of the fully probabilistic approach. The study of Richels et al. (2004) is an application of a more conditional probabilistic approach-as their results are made conditional to one major unknown, technology change (two sets of scenarios, one with optimistic technology change assumptions and one with pessimistic assumptions). The EMF-21 modeling study (Weyant et al., 2006) is an example of an application of the model comparison method to gain insight into uncertainty. The Millennium Assessment scenarios (MA) provide an example of the pure alternative scenario approach as based on a diverging storyline implemented by only one model for each topic these scenarios looked at (Carpenter and Pingali, 2006). The SRES report (Nakicenovic and Swart, 2000) combined two

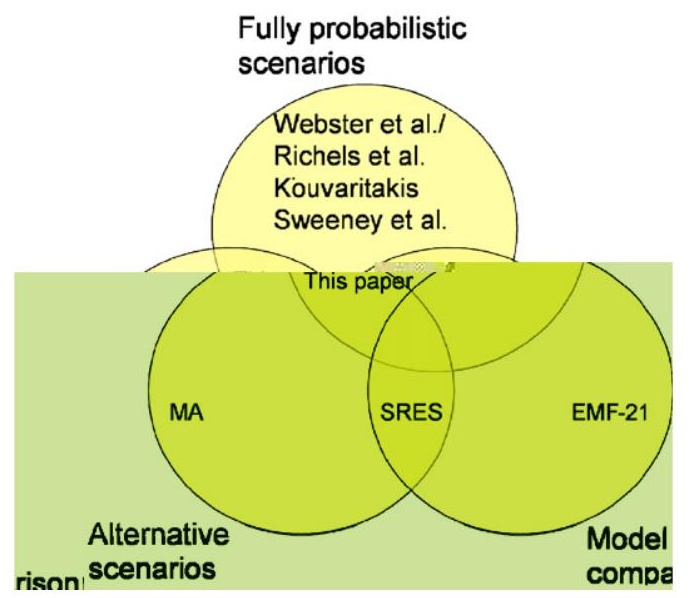

Fig. 11. Overview of earlier studies in comparison to the different methods for dealing with uncertainty in scenario analysis.

Table 3

Fossil fuel prices

\begin{tabular}{|c|c|c|c|c|c|c|c|c|c|}
\hline & \multicolumn{3}{|c|}{ Oil prices $(\$ / G J)$} & \multicolumn{3}{|c|}{ Gas prices $(\$ / G J)$} & \multicolumn{3}{|c|}{ Coal prices $(\$ / G J)$} \\
\hline & 2000 & 2050 & 2100 & 2000 & 2050 & 2100 & 2000 & 2050 & 2100 \\
\hline $\mathrm{A} 1$ & 3.7 & $6.6-9$ & $8.7-11.3$ & 2.2 & $5.4-8.0$ & $7.4-9.9$ & 1.1 & $1.4-1.5$ & $1.9-2.3$ \\
\hline A2 & 3.7 & $7.4-10.0$ & $10.6-14.9$ & 2.2 & $4.7-5.9$ & $8.5-10.8$ & 1.1 & $1.3-1.4$ & $2.3-3.2$ \\
\hline B1 & 3.7 & $6.1-8.7$ & $7.7-10.0$ & 2.2 & $4.3-6.3$ & $7.1-9.2$ & 1.1 & $1.3-1.4$ & $1.6-1.8$ \\
\hline B2 & 3.7 & $5.9-8.6$ & $8.3-10.8$ & 2.2 & $4.2-6.1$ & $7.7-9.9$ & 1.1 & $1.4-1.5$ & $2.0-2.3$ \\
\hline
\end{tabular}


approaches: development of 4/6 different storylines, but also comparison of the results of six different models.

Fig. 12 presents the outcomes of the studies indicated above for the cumulative and annual $\mathrm{CO}_{2}$ emissions in 2050 and 2100. The results, first of all, indicate that uncertainty increases in time in every single study. The ranges for each of the SRES scenarios in the original SRES report seem to be somewhat wider than the range developed here. There are two main explanations for this. First of all, the SRES range originates from the use of different models and hence also reflects model differences. For the A2 scenario, for instance, the high end of the range in SRES is represented by the ASF model that always shows relatively high coal consumption levels relative to other models, while the MARIA model shows high penetration rates of nuclear power resulting in relatively low emission levels (van der Sluijs et al., 2002). A second reason for the wider SRES range in the full range results of the A1 scenario is the explicit attention to the role of technology (A1T versus A1FI) (Nakicenovic and Swart, 2000). Although the sampling here allows for wide ranges of technology development rates and technology preferences, the resulting range still does not capture the one from the more explicit storyline approach taken in SRES.

On average, the scenarios of this study show slightly higher emissions than the corresponding IPCC-SRES scenarios. The reason for this is not obvious: new insights into population and income development, into fossil fuel resources and into 1995-2005 emission trajectories and model bias may all play a role. Only for B2, it is clear that some of the original SRES models have paid more attention to the "environmental orientation" of the original storyline. A model comparison study would be needed to gain more insight into the reason for higher $\mathrm{CO}_{2}$ emissions in this study vis-à-vis SRES for the other scenarios.

Comparing the results of this study to the fully probabilistic studies shows that the latter give both broader (Webster et al., 2002) and smaller range of outcomes (Richels et al., 2004; Sweeney et al., 2006) compared to the overall range of this study. The former is somewhat unexpected given the expectation that purely probability-based approaches may suffer from a bias towards one central set of assumptions. It should be noted, however, that the EPPA model used by Webster seems to be less constrained by inertia than TIMER: the lowest trajectories of Webster et al. (2002) show very low emissions in the first part of the century as a direct model response to certain assumptions. More recently, Webster and Cho (2006) concluded that the assumption of perfect correlation in economic growth rates among regions is also causing wider ranges in their analysis compared to a case where historically observed levels of correlation were accounted for.

The combination of the two ranges identified by Richels et al. (2004) roughly coincides with the range found here for the central B2 storyline. It should be noted that Richels et al. (2004) only vary a limited set of parameters in their analysis (population, GDP and technology assumptions) resulting in a narrower range. Comparison with the Sweeney et al. (2006) range leads to comparable outcomes-while the range of the modeling effort by Kouvaritakis and Panos (2005) (for 2050 only) shows the results of scenarios in this study to overlap well with their unconditional range.

Finally, we compare our results to the outcome of the EMF-21 study. The modelers participating in that study were all asked to
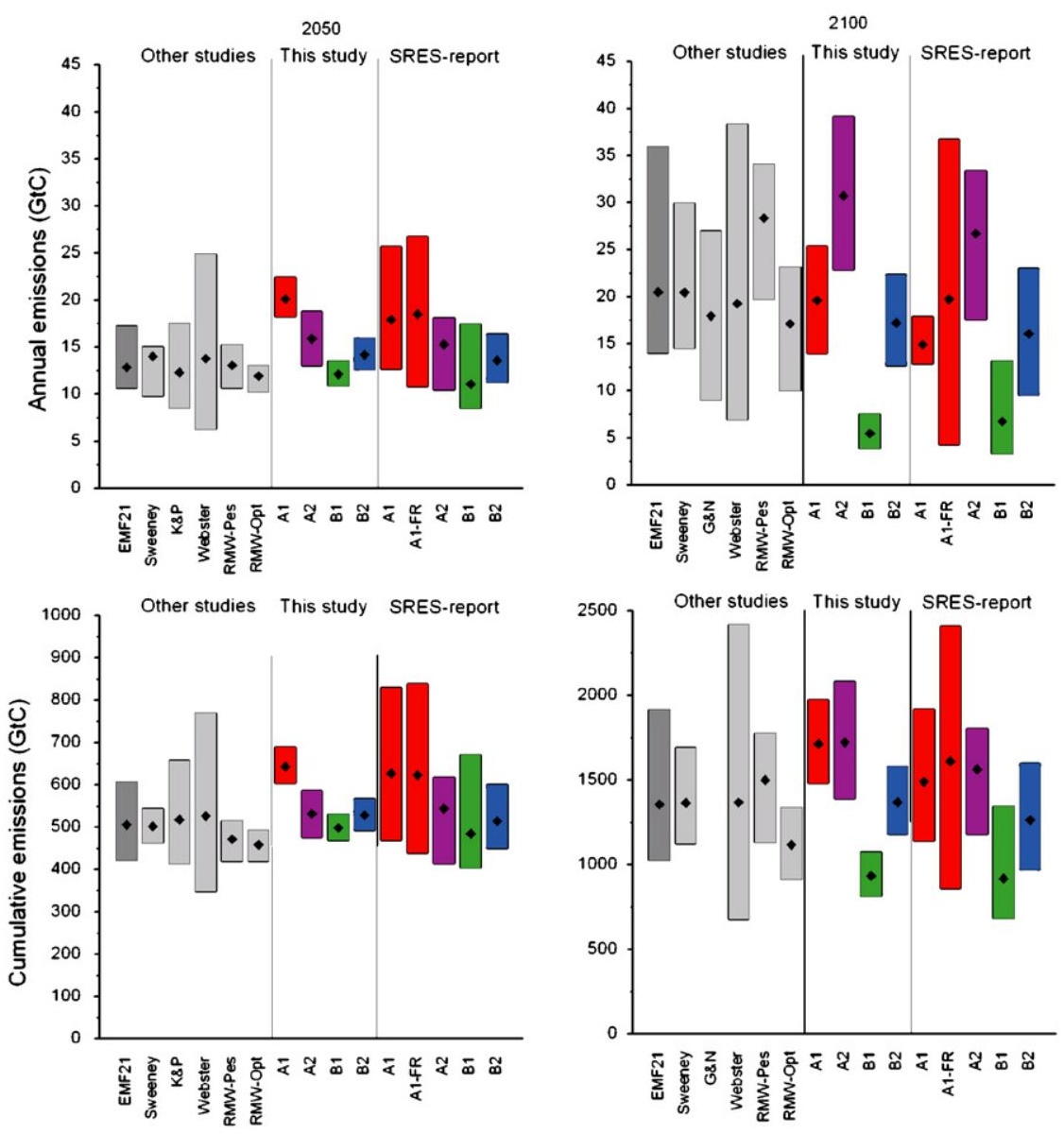

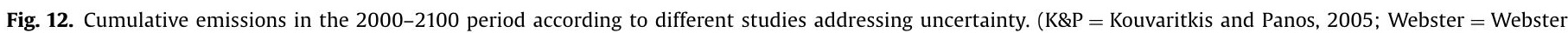
et al., 2002; RMW = Richels, Manne and Wigley, 2004). 
Table 4

Comparison of methods

\begin{tabular}{|c|c|c|}
\hline Uncertainty method & Indication of preferred application, strengths and weaknesses & $\begin{array}{l}\text { Type of uncertainties } \\
\text { typically addressed }\end{array}$ \\
\hline Full probabilistic analysis & $\begin{array}{l}\text { - Formal methodology } \\
\text { - Very suitable for dealing with statistical uncertainty } \\
\text { - Requires subjective assumptions on sampling ranges. }\end{array}$ & a, b1, 1 \\
\hline $\begin{array}{l}\text { Storyline-based } \\
\text { alternative scenarios }\end{array}$ & $\begin{array}{l}\text { - Very suitable for dealing with uncertainties originating from societal choice, value interpretation and } \\
\text { uncertainty or ignorance in relationships } \\
\text { - Able to construct consistent sets of coevolving variables. } \\
\text { - However, method requires many subjective, and often arbitrary choices. }\end{array}$ & b2, b3, c, d, 1, 3 \\
\hline Model comparison & $\begin{array}{l}\text { - Formal methodology } \\
\text { - Suitable for comparing uncertainty in formalized relationships or for } \\
\text { - Cotecting model bias } \\
\text { - Comparison often complex of many parameters and relationships are different across the models. }\end{array}$ & b, 2 \\
\hline $\begin{array}{l}\text { Conditional probabilistic } \\
\text { method }\end{array}$ & $\begin{array}{l}\text { - Allows for using the strengths of the storyline-based method (assumptions for not-easily quantifiable } \\
\text { parameters; consistent parameters choices) while also using statistical approaches. } \\
\text { - But requires considerable work and still arbitrary estimates for parameter estimates. }\end{array}$ & a, b, c, d, 1, 3 \\
\hline
\end{tabular}

contribute one single, modeler's preference baseline. In most cases, these baselines can be interpreted as the central-estimate scenarios of different modelers/models. The range across the EMF21 outcomes coincides reasonably with the B2 range of this study. The range is considerably narrower than the whole across all four scenarios of this study: neither the B1, nor the A2 range is represented, indicating that most modelers would not regard them as central baselines. ${ }^{4}$

The comparison of the studies as a whole provides some insight into the importance of different forms of uncertainty:

1. Uncertainty analysis within one particular model, as done here using the conditional probability approach may result in a similar range of outcomes, as generated by a multitude of models (such as EMF-21).

2. Fully probabilistic uncertainty analysis may result in ranges that are broader than those derived by storyline-based methods (Webster et al., 2002), but also result in more narrow ranges (2004). The differences between these studies show the role of subjective choices.

3. The uncertainty ranges generated by TIMER around the different storylines compare well to the ranges that are obtained by the other uncertainty studies.

An intriguing question remaining is what can be said about the total probability of the development of the 2000-2100 carbon emissions (the focus on this indicator comes from its relevance for long-term climate change). Some observations can be made on the basis of Fig. 12:

1. There is an overlap in the ranges of the A2, B2 and A1 scenarios in this study (between 1400 and $1600 \mathrm{GtC}$ ) despite the differences in storyline.

2. The fully probabilistic studies seem to show the strongest overlap in the $1100-1700 \mathrm{GtC}$ range (with the highest probabilities around $1400 \mathrm{GtC}$ ).

3. The modeler's preference baselines of EMF-21 range from 1000 to $1800 \mathrm{GtC}$ - with a central value of $1400 \mathrm{GtC}$.

\footnotetext{
${ }^{4}$ The EMF-21 study covers mainly economic models from the USA, Europe and Japan, possibly providing some bias in expectations.
}

Combined, scenario studies appear to obtain a majority of their results within a much more confined range than the total uncertainty range across all the different storylines. The question, however, remains: is this caused by collectively biased expectations with respect to the future-or does "the balance of evidence" suggest an indication of (now) likely emission levels, despite fundamental uncertainties? In this context, one may note that the full range of $\mathrm{B} 1$ and part of $\mathrm{A} 2$ is outside the ranges suggested here.

The analysis here is constrained to baseline (no climate policy) scenarios. A similar analysis can be done for mitigation scenarios, (1) either to identify probabilistic outcomes of scenarios conditional on both storyline and stabilization target (compare Webster et al. (2003) for a comparable analysis in the fully probabilistic approach), (2) or to identify strategies robust under different storylines (Groves and Lempert, 2007).

Based on the results of the analysis, and the deliberations that were made earlier, Table 4 represents an attempt to summarize some of the strengths and weaknesses of the various approaches.

\section{Conclusions}

- Conditional probabilistic scenario analysis can be used as a way to introduce statistical methods of uncertainty analysis, while recognizing deep uncertainties. Uncertainties represent a crucial element of scenario analysis. Two main methods are often presented as options for uncertainty analysis: the scenario approach and the fully probabilistic approach. This paper shows that it is possible to combine the two approaches (conditional probability analysis) in a way that allows formal analysis of those elements where meaningful probability estimates can be established, while still retaining the strong elements of a storyline approach to uncertainty. Storylines are a device for structured thinking about a future with deep uncertainty while also assumptions regarding the reasoning behind the choice of driving forces, parameter values, and modeling approaches are made more explicit. The added value of the conditional probabilistic approach compared to a nonconditional approach can also be observed from the analysis of most relevant uncertainties. These are shown to be a function of the storyline. Compared to the default alternative scenario method, the conditional probabilistic method (1) adds the 
strength of statistical methods in situations where they can meaningfully be applied, and (2) provides ranges for each scenario. The method, of course, also has also the limitations characteristic for the two methods it combines. In particular, it does not provide a single estimate of future emissions because no probabilities are assigned to the underlying storylines themselves. The method is also more elaborate than the default storyline-based alternative scenario method.

- The model calculations suggest that 21 st century cumulative emissions range from around 800 to $2500 \mathrm{GtC}$ in the absence of climate policy. The low end of the range originates in a different storyline than the high end of the range. The results indicate that $\mathrm{CO}_{2}$ emissions from the energy system may develop in very different directions, with emissions ranging from 4 to $40 \mathrm{GtC}$ in 2100 or in terms of cumulative 2000-2100 emissions, $800-2500 \mathrm{GtC}$. The reason for this wide range results partly from the fundamentally different way the 21 st century society could develop. The range found in this study is consistent with the range found in the SRES scenario study (from which the storylines used here are derived), but also with the range found in the fully probabilistic study of Webster et al. (2002). The smaller uncertainty ranges suggested by some other studies all coincide with the uncertainty range identified here for the socalled B2 world, based on a more-or-less business-as-usual type of storyline. As such, the conditional probabilistic approach can give one a sense of whether existing emissions scenarios are biased in a particular direction.

- Emissions for a clearly defined storyline could still include an uncertainty range of more than 40\%. These ranges originate from stochastic uncertainty and existing ambiguity in each storyline. Important variables contributing to this uncertainty are uncertainty in the development of driving forces such as population and income, uncertainty in energy efficiency improvement, oil resources, fuel preferences and technology development of biofuels and renewables. There seems to be a dominance of "energy demand"-related factors as causes of uncertainty. However, one needs to realize that in TIMER (just as in most other energy-system models) the supply sector is described with considerably more detail than the demand sector, and as a result the effects of single parameter values are smaller.

- There is considerable overlap in the uncertainty ranges identified for the A2, A1 and B2 storylines. The results for B1 stand out. Especially, the interpretation of the B2 scenario as a "medium" pathway, and the A1 storyline, results in a clear overlap of outcome ranges for several parameters. The B1 storyline, a normative choice for sustainable development and away from fossil fuels, produces very different results.

- The storylines explored here are deficient in many ways-and are therefore not likely to come true. For instance, the assumption of "no climate policy" is, given the current focus on climate change, highly unlikely. Moreover, the feedbacks of climate change to the drivers have not been considered. Similarly, the TIMER model also does not capture the possible feedbacks of the energy system on the economic drivers (e.g. of very high fossil fuels as a result of depletion). Finally, the scenarios are derived from caricature storylines that are continued over 100 years without surprises. Surprises, however, may occur, such as technology breakthroughs (fusion) or major wars. Furthermore, societies may shift from "one storyline to another".

\section{Acknowledgements}

The author would like to thank Brian O'Neill for discussions on conditional probabilistic methods and Arthur Petersen for suggestions on uncertainty qualification schemes.

\section{Appendix A. Storyline assumptions and assumed parameter ranges}

\section{A.1. Assumed ranges for driving forces}

The main (exogenous) driving forces of energy demand in TIMER are GDP growth, economic structure (here represented by share of industry in GDP) and population growth (Table A1).

1. Gross domestic product (GDP): In the model, energy demand for five sectors is driven by GDP or sectoral value added (see below).

2. Share of industry (\% of GDP): Energy demand in the industry sector is driven by industry value-added, in the service sector by service value-added. As energy intensity is generally lower in the service sector than in the industry sector, a shift in sectoral composition of GDP will influences energy demand.

3. Population: Population drives energy demand in all sectors.

We have analyzed the regional growth rates of four large global regions (as used in the IPCC-SRES report) for economic growth in the 1890-2000 period (based on 10-year averages in the

Table A1

Main storyline assumptions underlying the SRES scenarios

\begin{tabular}{|c|c|c|c|c|c|c|}
\hline & \multicolumn{3}{|l|}{ A1 } & $\mathrm{A} 2$ & B1 & B2 \\
\hline Storyline & \multicolumn{3}{|c|}{ Globalization; liberalization } & $\begin{array}{l}\text { Heterogeneous world; self- } \\
\text { reliance; fragmentation }\end{array}$ & $\begin{array}{l}\text { Globalization; orientation on social } \\
\text { and environmental sustainability }\end{array}$ & $\begin{array}{l}\text { Local solutions to } \\
\text { sustainability; regional } \\
\text { emphasis }\end{array}$ \\
\hline Population & \multicolumn{3}{|l|}{ Low } & High & Low & Medium \\
\hline Economic growth & \multicolumn{3}{|l|}{ Very high } & $\begin{array}{l}\text { Low in developing countries; } \\
\text { medium in industrialized } \\
\text { countries }\end{array}$ & High & Medium \\
\hline $\begin{array}{l}\text { Attitude towards } \\
\text { environmental } \\
\text { protection }\end{array}$ & \multicolumn{3}{|l|}{ Reactive } & Reactive & Proactive & Proactive \\
\hline $\begin{array}{l}\text { Main goal for the } \\
\text { energy system }\end{array}$ & \multicolumn{3}{|c|}{ Reliable, cheap energy for everybody } & Security of energy supply & $\begin{array}{l}\text { Energy services within sustainable } \\
\text { limits }\end{array}$ & $\begin{array}{l}\text { Combination of different } \\
\text { goals }\end{array}$ \\
\hline Primary energy use & \multicolumn{3}{|l|}{ Very high } & High & Low & Medium \\
\hline $\begin{array}{l}\text { Technology } \\
\text { development }\end{array}$ & \multicolumn{3}{|l|}{ Rapid } & Slow & Rapid & Medium \\
\hline $\begin{array}{l}\text { Type of technology } \\
\text { development }\end{array}$ & $\begin{array}{l}\text { Balanced } \\
\text { (A1B) }\end{array}$ & $\begin{array}{l}\text { Primarily } \\
\text { fossil fuels } \\
\text { (A1FI) }\end{array}$ & $\begin{array}{l}\text { Primarily non- } \\
\text { fossil energy } \\
\text { (A1T) }\end{array}$ & Balanced & $\begin{array}{l}\text { Primarily energy efficiency and non- } \\
\text { fossil energy }\end{array}$ & Balanced \\
\hline
\end{tabular}


Table A2

Description of sampling ranges for driving forces

\begin{tabular}{|c|c|c|c|c|c|}
\hline & A1 & A2 & B1 & B2 & Rationale \\
\hline \multicolumn{6}{|c|}{ GDP (\% growth in constant\$ in the $2000-2100$ period) } \\
\hline Default values & 2.7 & 1.2 & 2.3 & 2.2 & $\begin{array}{l}\text { Here global values are shown. However, in reality we use } \\
\text { regionally defined growth rates consistent with the IMAGE } 2.3 \\
\text { implementation of the IPCC-SRES scenarios. }\end{array}$ \\
\hline Sample ranges & $2.4-3.2$ & $1.0-1.5$ & $2.0-2.7$ & $1.6-2.4$ & Regionally defined ranges based on the historically founded values \\
\hline \multicolumn{6}{|l|}{ Share industry (\% of GDP in 2100) } \\
\hline Default values (\% of total GDP) & 0.36 & 0.35 & 0.27 & 0.37 & $\begin{array}{l}\text { Based on the IMAGE } 2.3 \text { implementation of the SRES scenarios and } \\
\text { underlying WorldScan calculations (IMAGE-team 2001) }\end{array}$ \\
\hline Sample ranges & $0.32-0.40$ & $0.31-0.39$ & $0.24-0.31$ & $0.33-0.41$ & $\begin{array}{l}0.04 \text { used on the basis of current variation among OECD regions } \\
\text { ( } 15 \% \text { range in total) }\end{array}$ \\
\hline \multicolumn{6}{|c|}{ Population in 2050 and 2100 (billion) } \\
\hline Default values & $8.2 / 6.9$ & $10.4 / 12.5$ & $8.2 / 6.9$ & $9.0 / 9.1$ & Both default values and ranges are based on O'Neill (2004) \\
\hline \multirow[t]{2}{*}{ Sample ranges } & $7.6-8.6 /$ & $8.5-13.7$ & $7.6-8.6 /$ & $8.3-10$ & \\
\hline & $5.6-8.2$ & $9.2-16.0$ & $5.8-8.0$ & $7.5-10.8$ & \\
\hline
\end{tabular}

1890-1970 period on the basis of HYDE data). Furthermore, we studied the 5-year moving average for the 1970-2000 period (based on the World Bank Development Indicators). For the OECD region, a normal distribution was found-with an average per capita growth of $2.2 \%$ and a $95 \%$ range from $1.2 \%$ to $3 \%$. The other three regions (Central Europe and the Former Soviet Union (REF), Asia (ASIA) and Africa-Latin America-Middle East region (ALM)) had much wider historical ranges with distinct temporal patterns. For Asia, growth rates were found mostly in a $0-1 \%$ range during the $1890-1970$ period and a $4-6 \%$ range in the $1970-2000$ period (after the "take-off" phase of some of Asia's economies). A broad range was also found in the ALM region, but with almost an opposite temporal distribution.

Based on the historical distributions, we could propose regionally defined economic growth rates and their distributions for each region, depending on the four storylines-with the mean values roughly consistent with the IMAGE 2.3 implementation of the IPCC scenarios (see Table A2). It should be noted that using the 5-10-year growth values as indicative for the uncertainty in longterm growth pattern, the resulting 100-year growth level for the highest (A1) and lowest (A2) storylines are considerable higher and lower, respectively, than the growth rates that have actually occurred in the past over such a long time period.

For economic structure, the size of the industrial sector plays an important role as it is the most energy-intensive sector. The central values (by region as a function of time) were set on the basis of the IMAGE implementation of the IPCC-SRES scenarios (IMAGE-team, 2001), in turn, based on the runs of Bollen (2004). Analysis shows the current variation among OECD regions for the relative size of the industry sector (compared to GDP) to be around $15 \%$. On this basis a conditional sampling range of $8 \%$ ( $4 \%$ above and below the central value) was assumed.

Finally, for population O'Neill (2004) published a set of scenarios conditional to the SRES storylines. We took the 95\% intervals from this publication, and sampled within these ranges, assuming normal distribution. The assumption of normal distribution is reasonably consistent with the distributions reported by O'Neill.

\section{A.2. Assumed ranges for factors determining energy demand}

In addition to the driving forces discussed above, several other factors determine energy demand: these include AEEI, PIEEI and structural change (SC) within sectors.

1. AEEI captures forms of efficiency improvement not caused by price changes but general technology improvement. For example, the presence of more efficient boilers at the time an old boiler is replaced.

2. PIEEI: This factor describes the impact of increasing prices on energy efficiency.

3. SC: This factor describes the energy intensity development within sectors independent of efficiency improvement (e.g. transport modes).

In TIMER, AEEI is assumed to relate to GDP growth (Table A3) in a similar way as described by Richels et al. (2004), although we also assume that this percentage declines over time as a result of (slowly) approaching thermodynamic limits. Interpreting the variation (unconditional range) applied by Webster et al. (2002) (0.25-1.5\% annually for OECD countries) means that the samples mostly $25 \%$ in either direction relative to his economic growth rates. Given no other inputs on this parameter, we have assumed these numbers to form the basis of our ranges.

The contribution of price-induced energy efficiency improvement in TIMER depends mainly on the assumed pay-back time. We applied a variation of $15 \%$ to these values-based on the default assumptions made in each scenario and the requirement to keep the scenarios sufficiently distinct.

Finally, structural change by TIMER captures changes in the type of activities over time within each sector (e.g. shifts from heavy to light industry). The TIMER description assumes a longterm saturation of energy demand per sector (in terms of GJ per capita). In the scenarios, one factor is used to scale this saturation up/downward as a function of time based on the storyline of the scenario. This factor reflects the emphasis on energy-intensive services in the scenario and is used here for uncertainty analysis. To assess its potential range, we analyzed the differences in per capita energy consumption of the different representations of the SRES scenario per storyline (Nakicenovic and Swart, 2000). Values of $30-50 \%$ variation among the central values were generally found for different model representations of the same storyline. Assuming this to a reasonable indication of the uncertainty range, we used a sampling range of $15 \%$ upwards and downwards.

\section{A.2.1. Technology change}

Technology is represented in TIMER both by learning curves (progress as a function of cumulative experience) and timedependent exogenous inputs. We have clustered the technology variables into different groups: learning curves for (1) fossil fuel production, (2) renewables in the power sector, (3) nuclear power, (4) bio-energy and (5) energy demand, (6) hydrogen technologies and time-dependent assumptions for (7) thermal power plants. The learning curves are a function of the so-called progress ratio. 
- Progress ratio: A measure of improvement for a doubling of experience, where a value of 0.8 indicates a $20 \%$ improvement for each doubling.

Assessments of the historical pdf have been made for technology in general (Argotte and Epple, 1990) and energy technology in particular (McDonald and Schrattenholzer, 2002). The results of these studies tend to reveal wide ranges - with most values found between 0.7 and 1.0. Progress ratios in TIMER are dependent on technology, time and scenario. Taking the conditional range to be half the unconditional uncertainty range $(0.3)$, we have samples for each scenario with a value of 0.07 above and below the default values. Sampling was done independently for the clusters of technologies mentioned above. For thermal power technologies, upward and downward sampling of $4 \%$ was applied on the basis of the variation across the different scenarios (Table A4).

\section{A.2.2. Resources}

For fossil fuel resources, standard values in TIMER are based on those reported by Mulders et al. (2006) using the methodology of Rogner (1997). For each fossil fuel, Rogner provides different categories varying in production costs and probability of occurrence (each category assumed to have higher production costs than the previous). Together, these categories form a long-term supply-cost curve for oil, natural gas and coal. For conventional resources of oil and gas, the Mulders et al. numbers (categories 1-4) are based on the USGS estimates for the reserves and resources, with a different likelihood of occurrence (costs estimates added by Rogner).

1. Resources of fossil fuels: Available amounts of oil, natural gas and coal per costs category.
2. Renewable resources: Maximum use by category of renewable energy; in TIMER the form of the supply-cost curve is kept constant.

In our analysis, we assumed these estimates to be independent of the storyline and were able to assign probability values to each of these categories in such way that the total probability of these categories collectively again reflected the original USGS probability estimate for total conventional oil and gas resources. This results in a range of conventional oil resources of $7-17 \mathrm{ZJ}$. Interestingly, the lower end of this range equals estimates provided by the proponents of the "end-of-cheap-oil" hypothesis (Laherre and Cambell, 1999). In other words, in most of our probabilistic runs we included substantially higher resource estimates than the peak-oil proponents but our runs do not preclude their estimates.

For unconventional resources of oil and gas and for coal, probability ranges are much harder to derive as no concrete ranges were found in the literature. For unconventional gas resources, for instance, ranges provided in the literature seem to have more relevance for geology than for energy production. In contrast to conventional resources, the values provided by Rogner do not represent the upper range, but best-guess estimates. Therefore for unconventional oil, we assumed a rather arbitrary range of $50 \%$ around Rogner's estimates, while for gas, we assumed a range of $70 \%$ relative to Rogner's estimates. The higher number for natural gas comes from the fact that here unconventional resources represent mainly gas hydrates, an enormous source of potential energy but characterized by a huge uncertainty with respect to the potential use of natural gas. For coal, Rogner's estimates represent best-guess values for each category. We applied a sampling range, both upwards and downwards, of around $25 \%$.

Table A3

Description of sampling ranges for parameters determining energy demand

\begin{tabular}{|c|c|c|c|c|c|}
\hline & A1 & A2 & B1 & $\mathrm{B} 2$ & Rationale \\
\hline \multicolumn{6}{|c|}{ AEEI (as \% of GDP per capita growth) } \\
\hline Default values & \multicolumn{4}{|c|}{$\begin{array}{l}0.28-0.44 \% \text { of GDP per capita growth } \\
\text { (depending on region and sector) }\end{array}$} & \\
\hline Sample ranges & $\pm 25 \%$ & $\pm 25 \%$ & $\pm 25 \%$ & $\pm 25 \%$ & Based on the variation applied by Webster et al. \\
\hline \multicolumn{6}{|c|}{ Accepted pay-back times (years) } \\
\hline Default values & 3.4 & 2.8 & 6 & 3.2 & Industry sector; similar trends for other sectors \\
\hline Sample ranges (\%) & \pm 15 & \pm 15 & \pm 15 & \pm 15 & Based on the assumed default values \\
\hline \multicolumn{6}{|c|}{ Structural change (2100 multiplication on energy demand compared to standard TIMER setting) } \\
\hline Default values & 1.75 & 1.50 & 0.85 & 1.25 & $\begin{array}{l}\text { A1 is representative of a saturation of per capita energy use (at high income and temperate } \\
\text { zones) of } 20-30 \% \text { above US levels; B1 is found } 30 \% \text { below US levels }\end{array}$ \\
\hline Sample ranges (\%) & \pm 15 & \pm 15 & \pm 15 & \pm 15 & $\begin{array}{l}\text { The proposed range complies with the general rule assuming that the B1-A } 1 \text { range is } \\
\text { representative of the full uncertainty range. The range between differences per capita energy } \\
\text { use of the same scenario as reported by different models in SRES report is also around } 30-50 \%\end{array}$ \\
\hline
\end{tabular}

Table A4

Description of sampling ranges for parameters determining technology progress

\begin{tabular}{|c|c|c|c|c|c|}
\hline & A1 & A2 & B1 & B2 & Rationale \\
\hline \multicolumn{6}{|l|}{ Progress ratios } \\
\hline Default values & $0.7-1.05$ & $0.7-1.05$ & $0.7-1.05$ & $0.7-1.05$ & $\begin{array}{l}\text { Range captures all values as function of time, technology and } \\
\text { storyline }\end{array}$ \\
\hline Sample ranges & \pm 0.07 & \pm 0.07 & \pm 0.07 & \pm 0.07 & $\begin{array}{l}\text { This represents about } 25 \% \text { of the unconditional range in } p \text {-values } \\
\text { found in the literature (Argotte and Epple, 1990; McDonald and } \\
\text { Schrattenholzer, 2002) }\end{array}$ \\
\hline \multicolumn{6}{|c|}{ Efficiency of thermal power plants } \\
\hline Sample ranges & \pm 0.04 & \pm 0.04 & \pm 0.04 & \pm 0.04 & $\begin{array}{l}\text { Sampling based on the assumed variation across the differences } \\
\text { scenarios }\end{array}$ \\
\hline
\end{tabular}


A wide range of estimates for potentials can also be found for renewables. De Vries et al. (2007) recently provided an estimate of storyline-based long-term costs supply-cost curves that have also been used as input for the IMAGE 2.3 scenarios. De 
3. Capacity credit: The capacity value assigned to renewables is assumed to decline with increasing renewable penetration. The shape of this curve can be influenced by the credit factor.

4. Energy taxes: Taxes on top of energy prices as function of sector and region.

5. Short-term uncertainty in oil/gas prices: A factor added to the model to reflect factors influencing oil and gas prices outside the scope of the model. This factor ensures that the oil price is set at a level of $50-60 \$ /$ bbl in 2005 .

The fuel preference values were varied in the analysis by $50 \%$ for each scenario. Since no external information was available, the range was based on the variation in values in the historical calibration and across different scenarios.

The added value on transport costs, reflecting trade barriers, were varied by $50 \%$ in either direction in our probabilistic modeling. Again, the range is based on their values in the original scenarios.

An important factor for the penetration of intermittent renewables into the electric power system is the assigned capacity credit as a function of penetration. On the basis of various curves published in the literature (see Giebel, 2005), we have shifted the curve used in TIMER with a factor of 2 upward and downward.

For secondary energy taxes, values in the scenarios were based on current values in different regions. In the uncertainty analysis these levels were varied by $50 \%$, based on the existing differences between the scenarios and current regional variation.

Finally, present-day oil and natural gas prices in TIMER can only be represented by an assumption that other factors-longterm supply-cost curves and simple price-setting equations-have a substantial influence on fossil fuel prices (the equilibrium price of oil in TIMER is around $25 \mathrm{US} \$ / \mathrm{bbl}$ ). Important factors that currently contribute to high oil prices and which are not represented in the model are lack of production capacity, speculation and supply insecurity. As it is uncertain how long these factors will continue to determine oil prices, the short- to medium-term price increase has been added as an additional uncertainty. This factor is defined by the year that prices return to equilibrium, assuming a linear decrease (varying from 2008 to 2050). The gas price is assumed to be coupled to the oil price (Table A6).

\section{References}

Argotte, L., Epple, D., 1990. Learning curves in manufacturing. Science 247, 920-924.

Bollen, J.C., 2004. A Trade View on Climate Change Policies, A Multi-Region MultiSector Approach. University of Amsterdam.

Carpenter, S., Pingali, P., 2006. Ecosystems and Human Wellbeing: Scenarios. Island Press, Washington, DC, USA.

De Vries, H.J.M. (Ed.), 2006. Scenarios Guidance for an Uncertain and Complex World? MIT Press, Cambridge.

De Vries, H.J.M., Hoogwijk, M., van Vuuren, D.P., 2007. The potential supply of renewable energy: wind, solar and bio-energy. Energy Policy 35 (4), 2590-2610.

Deffeyes, K., 2006. Hubbert's Peak. The Impending Oil Shortage. Princeton University Press, Princeton, NJ.

Dessai, S., Hulme, M., 2001. Climatic implications of revised IPCC emissions scenarios, the Kyoto protocol and quantification of uncertainties. Integrated Assessment 2 (3), 159-170.

Dessai, S., Hulme, M., 2004. Does climate adaptation policy need probabilities? Climate Policy 4, 107-128.

Dessai, S., O’Brien, K., Hulme, M. (Eds.), 2007. Editorial: on uncertainty and climate change, Global Environmental Change 17, 1-3.

Giebel, G., 2005. Wind Power has a Capacity Credit a Catalogue of 50+ Supporting Studies, Risø National Laboratory.

Groves, D.G., Lempert, R.J., 2007. A new analytic method for finding policy-relevant scenarios. Global Environmental Change 17, 73-85.

Grübler, A., Nakicenovic, N., 2001. Identifying dangers in an uncertain climate. Nature 412,15

Grübler, A., O'Neill, B., Van Vuuren, D., 2006. Avoiding hazards of best-guess climate scenarios. Nature 440 (7085), 740.
Hulme, M., Carter, T., 1999. Representing uncertainty in climate change scenarios and impact studies. In: Carter, T., Hulme, M., Viner, D. (Eds.), ECLAT-2 Workshop Report. Climatic Research Unit, Norwich.

IMAGE-team, 2001. The IMAGE 2.2 implementation of the IPCC SRES scenarios. A comprehensive analysis of emissions, climate change and impacts in the 21st century. Report No. RIVM CD-ROM publication 481508018, National Institute for Public Health and the Environment, Bilthoven, the Netherlands.

Kouvaritakis, N., Panos, V., 2005. Uncertainty Analysis of Energy and Climate Change Outlooks Developed for the 2005 Report, E3M-lab, National Technical University of Athens, Athens.

Laherre, J., Cambell, C., 1999. The end of cheap oil. Scientific American.

Leggett, J., Pepper, W.J., Swart, R.J., Edmonds, J., Meira Filho, L.G., Mintzer, I., Wang, M.X., sjkdfhjks, J.W., 1992. Emissions scenarios for the IPCC: an update. In: Climate Change 1992: The Supplementary Report to The IPCC Scientific Assessment. Cambridge University Press, Cambridge, UK, pp. 68-95.

Lempert, R., Nakicenovic, N., Sarewitz, D., Schlesinger, M., 2004. Characterizing climate-change uncertainties for decision-makers. Climatic Change 65, 1-9.

Manne, A.S., Richels, R.G., 1994. The costs of stabilizing global $\mathrm{CO}_{2}$ emissions: a probabilistic analysis based on expert judgments. The Energy Journal 15 (1), 31-56.

McDonald, A., Schrattenholzer, L., 2002. Learning curves and technology assessment. International Journal of Technology Management 23 (7/8), 718-745.

Moss, R.H., Schneider, S.H. (Eds.), 2000. Uncertainties in the IPCC TAR: Recommendations to Lead Authors for More Consistent Assessment and Reporting. World Meteorological Organization, Geneva.

Mulders, F.M.M., Hettelar, J.M.M., Van Bergen, F., 2006. Assessment of the Global Fossil Fuel Reserves and Resources for TIMER. TNO Built Environment and Geosciences, Utrecht, The Netherlands.

Nakicenovic, N., Swart, R. (Eds.), 2000. Special Report on Emissions Scenarios (SRES). Cambridge University Press, Cambridge, UK.

Nordhaus, W.D., Popp, D., 1997. What is the value of scientific knowledge? An application to global warming using the PRICE model. The Energy Journal 18 (1), $1-45$.

NRC, 1996. Understanding Risk: Informing Decisions in a Democratic Society. National Academy Press, Washington, DC.

O'Neill, B.C., 2004. Conditional probabilistic population projections: an application to climate change. International Statistical Review 72 (2), 167-184.

O'Neill, B.C., 2005. Population scenarios based on probabilistic projections: an application for the millennium ecosystem assessment. Population and Environment 26 (3), 229-254.

Patt, A., 2007. Assessing model-based and conflict-based uncertainty. Global Environmental Change 17, 37-46.

Patt, A., Dessai, S., 2005. Communicating uncertainty: lessons learned and suggestions for climate change assessment. Comptes Rendus Geoscience 337, 425-441.

Pepper, W., Sankovski, A., Leggett, J., 2005. Probabilistic modeling of sulfur and nitrogen pollution controls and their relations with income. Journal of Environment \& Development 14 (1), 197-219.

Petersen, A.C., 2006. Simulating nature: a philosophical study of computersimulation uncertainties and their role in climate science and policy advice. Ph.D. Dissertation, Vrije Universiteit Amsterdam.

Richels, R.G., Manne, A.S., Wigley, T.M.L., 2004. Moving beyond concentrations: the challenge of limiting temperature change. Report no. Working paper 04-11, AEI-Brooking Joint Center for Regulatory Studies, Washington, DC.

Rogner, H.H., 1997. An assessment of world hydrocarbon resources. Annual Review of Energy and the Environment 22, 217-262.

Rotmans, J., de Vries, H.J.M., 1997. Perspectives on Global Change: The TARGETS approach. Cambridge University Press, Cambridge, UK.

Saltelli, A., Chan, K., Scott, E.M., 2000. Sensitivity Analysis. Wiley, Chichester.

Saltelli, A., Tarantola, S., Campolongo, F., Ratto, M., 2004. Sensitivity Analysis in Practice. A Guide to Assessing Scientific Models. Wiley, Chichester

Schneider, S.H., 2001. What is "Dangerous" climate change? Nature 411, 17-19.

Schneider, S.H. (Ed.), 2002. Can we estimate the likelihood of climatic changes at 2100? An Editorial Comment, Climatic Change 52, 441-451.

Scott, M.J., Sands, R.D., Edmonds, J., Liebetrau, A.M., Engel, D.W., 1999. Uncertainty in integrated assessment models: modeling with MiniCAM 1.0. Energy Policy 27 (14), 597.

Sweeney, J., Weyant, J., Bhattacharjya, D., Blanford, G., Calvin, K., Eom, J., Gillingham, K., Hong, T., Mascarenhas, O., Mokriam, P., Sharma, D., 2006. Integrated Assessment of Energy Technologies: An Overview, Stanford University, Stanford.

van der Sluijs, J.P., Potting, J., Risbey, J., van Vuuren, D.P., de Vries, B., Beusen, A., Heuberger, P., Corral Quintana, S., Funtowicz, S., Kloprogge, P., Nuijten, D., Petersen, A., Ravetz, J., 2002. Uncertainty Assessment of the IMAGE/TIMER B1 $\mathrm{CO}_{2}$ Emissions Scenario, using the NUSAP Method, Dutch National Research Program on Climate Change, Bilthoven.

Van der Sluijs, J.P., Risbey, J.S., Kloprogge, P., Ravetz, J.R., Funtowicz, S.O., Corral Quintana, S., Guimaraes Pereira, A., De Marchi, B., Petersen, A.C., Janssen, P.H.M., Hoppe, R., Huijs, S.W.F., 2003. RIVM/MNP guidance for uncertainty assessment and communication. Detailed Guidance, Utrecht University, Utrecht.

Van Vuuren, D.P., 2007. Energy systems and climate policy: long-term scenarios for an uncertain future. Ph.D. Thesis, Utrecht University.

van Vuuren, D.P., O'Neill, B.C., 2006. The consistency of IPCC's SRES scenarios to 1990-2000 trends and recent projections. Climatic Change 75 (1-2), 9-46. 
van Vuuren, D.P., van Ruijven, B., Hoogwijk, M., Isaac, M., De Vries, B., 2006a. TIMER-2-Model description and application. In: Bouwman, L., Kram, T., KleinGoldewijk, K. (Eds.), IMAGE 2.4 Model Description. MNP-Netherlands Environmental Assessment Agency, Bilthoven.

van Vuuren, D.P., Weyant, J., De la Chesnaye, F., 2006b. Multigas scenarios to stabilise radiative forcing. Energy Economics 28 (1), 102-120.

van Vuuren, D.P., Den Elzen, M.G.J., Lucas, P., Eickhout, B.E., Strengers, B.J., Van Ruijven, B., Wonink, S., Van Houdt, R., 2007. Stabilizing greenhouse gas concentrations at low levels: an assessment of reduction strategies and costs. Climatic Change 81 (2), 119-159.

Webster, M., Cho, C.-H., 2006. Analysis of variability and correlation in long-term economic growth rates. Energy Economics 28, 653-666.
Webster, M.D., Babiker, M.H., Mayer, M., Reilly, J.M., Harnisch, J., Hyman, R., Sarofim, M.C., Wang, C., 2002. Uncertainty in emissions projections for climate models. Atmospheric Environment 36 (22), 3659-3670.

Webster, M.D., Forest, C., Reilly, J.M., Babiker, M., Kickligher, D., Mayer, M. Prinn, R., Sarofim, M.C., Sokolov, A., Stone, P., Wang, C., 2003. Uncertainty analysis of climate change and policy response. Climatic Change 61, 295-320.

Weyant, J.P., de la Chesnaye, F.C., Blanford, G.J., 2006. Overview of EMF-21: multigas mitigation and climate policy. The Energy Journal (special issue).

Wigley, T.M.L., Raper, S.C.B., 2001. Interpretation of high projections for globalmean warming. Science 293, 451-454. 